\title{
Virtual engineering at work: the challenges for designing mechatronic products
}

\author{
Herman Van der Auweraer · Jan Anthonis • \\ Stijn De Bruyne $\cdot$ Jan Leuridan
}

Received: 24 February 2011/ Accepted: 22 August 2012/Published online: 28 September 2012

(C) The Author(s) 2012. This article is published with open access at Springerlink.com

\begin{abstract}
The product race has become an innovation race, reconciling challenges of branding, performance, time to market and competitive pricing while complying with ecological, safety and legislation constraints. The answer lies in "smart" products of high complexity, relying on heterogeneous technologies and involving active components. To keep pace with this evolution and further accelerate the design cycle, the design engineering process must be rethought. The paper presents a mechatronic simulation approach to achieve this goal. The starting point is the current virtual prototyping paradigm that is widely adopted and that continues to improve in terms of model complexity, accuracy, robustness and automated optimization. Two evolutions are discussed. A first one is the extension to multi-physics simulation answering the design needs of the inherent multi-disciplinarity of "intelligent" products. Integration of thermal, hydraulic, mechanical, haptic and electrical functions requires simulation to extend beyond the traditional CAD-FEM approach, supporting the use of system, functional and perception models. The second evolution is the integration of control functions in the products. Where current industrial practice treats mechanical system design and control design as different design loops, this paper discusses their integration in a modelbased design process at all design stages, turning concepts such as software-in-the-loop and hardware-in-the-loop into basic elements of an industrial design approach. These concepts are illustrated by a number of automotive design engineering cases, which demonstrate that the combined
\end{abstract}

H. Van der Auweraer $(\bowtie) \cdot J$. Anthonis · S. De Bruyne . J. Leuridan

LMS International, Interleuvenlaan 68, 3001 Leuven, Belgium

e-mail: herman.vanderauweraer@1msintl.com

URL: http://www.lmsintl.com use of perception, geometric and system models allows to develop innovative solutions for the active safety, lowemission and high-comfort performance of next-generation vehicles. This process in turn poses new challenges to the design in terms of the specification and validation of such innovative products, including their failure modes and fault-tolerant behaviour. This will imply adopting a modelbased system engineering approach that is currently already common practice in software engineering.

Keywords Mechatronic systems - Design engineering · CAE $\cdot$ Control $\cdot$ Multi-disciplinary

\section{Introduction}

Product innovation managers face continuously increasing challenges with respect to their product portfolio. The traditional demands for improved performance, time to market and competitive price setting are strained by requirements related to product branding, personalization and ecological, safety and legislation aspects. This leads to increasingly complex, "mechatronic", products relying on active components and implemented by heterogeneous technologies.

Integrated design and engineering methods based on physical and virtual testing have become standard practices in the product design process. Extending these methods to support the development of mechatronic products requires addressing the challenges posed by (1) their inherent multidisciplinarity and (2) the integration of control system concepts.

The first challenge relates to the fact that nearly all simulation tools that have been deployed over the past 20 years to support product design engineering (finite 
element analysis, multi-body simulation...) are driven from geometry, styling preceding engineering. Integrating hydraulic, electronic, electromechanical and other complex functions is essentially done by independent design tasks, requiring the use of extra non-geometric simulation methodologies. The basic geometric design choices act as a major constraint for these tasks and going back to iterating the geometry is very difficult and leads to large delays. Frontloading the design engineering process hence requires adoption of simulation methods that extend beyond the traditional CAD-driven approach and that support the use of system and functional models crossing the boundaries of a wide range of disciplines, allowing to decide on system architectures before the geometry is available $[1,2]$.

The second challenge relates to integrating systems and control engineering. Currently, subsystems with active functions are treated as add-ons developed independently from the basic mechanical system. Suboptimal designs, unexpected integration problems and unexploited synergetic effects are the result $[3,4]$. Addressing this challenge requires bringing together the systems and the control design, across all phases of the design process (from system target setting over component development and testing to system integration). This leads to new physical and virtual testing paradigms such as software-in-the-loop, model-in-the-loop and hardware-in-the-loop as cornerstones for integrated intelligent system design engineering. This approach to system design can best be described by the model-based system engineering paradigm, known from the software world and increasingly considered as the way forward in general product design engineering [5-9].

\section{Automotive industry challenges}

The automotive industry represents a significant part of the economic activity, in Europe and globally. Innovation drivers are the improvement of customer satisfaction (performance, fuel/energy consumption, personalization, safety, comfort, brand values,...) and the adherence to increasingly strict environmental and safety regulations, while at the same time reducing design and manufacturing costs and the time to market. More new vehicle concepts, new vehicle architectures and functions are designed than ever before.

A fundamental evolution that is taking place in this industry is the increase of the electronic and mechatronic content in vehicles. Several studies estimate that the related increase to the vehicle value has risen to $40 \%$ in 2010 and that up to $80 \%$ of the automotive innovation will come from intelligent systems [10-13]. This of course relates in part to entertainment and telematics systems, but also to the use of many control systems applied to power train, chassis and body engineering [14-16]. One example is the optimization of performance, economy and emissions with engine and transmission controls to realize "green" driving through energy regeneration, automatic start/stop and smart driving control. Another example is the realization of "safe" driving, through the application of ABS (antilocking brake systems) and ESC (electronic stability control) systems for vehicle dynamics control, but also through the adoption of numerous advanced driver assistance systems (ADAS), e.g. for lane departure, active cruise control, object and pedestrian detection and many more to come in the next few years. Furthermore, every vehicle design has to aim ultimately at best customer experience, using control systems to optimize the ride comfort, handling behaviour and driveability.

This evolution impacts not only the vehicle product content itself, but also the way the vehicle design and development process has to change to enable widespread market introduction in standard vehicles [11, 13, 17, 18]. In the traditional approach, the mechatronic vehicle innovations remain on the level of add-on systems and a major need exists to integrate all functionality on the vehicle level through a systems approach. Configuration and performance optimization, system integration, control, component, subsystem and system-level validation of the intelligent systems must become an intrinsic part of the standard vehicle engineering process, just as this is today the case for the structural, vibro-acoustic and kinematic design. It is demonstrated that the discussed mechatronic simulation approach contributes to achieving this goal by providing solutions on both levels: multi-physics simulation and control engineering integration.

\section{Engineering challenges for mechatronic vehicle systems}

In a mechatronic system, the mechanical, electrical, thermal... components of a product are connected through sensors and actuators with controllers that define the overall functioning. The performance engineering of such mechatronic products hence mandates simulation and test solutions that are capable of analysing and optimizing the performance of such a product, taking into account (1) the interactions of components and subsystems in the product, each with their different physics representations (mechanical, thermal, fluids...) and (2) working as "active" systems, with sensors and actuators, and interconnected to controllers.

This requires the combined simulation of multi-physics systems and the controls, e.g. simulating vehicle dynamics with ESC. This is also why test systems that are used to describe and troubleshoot physical designs and validate 
Fig. 1 Double-V process for mechatronic systems

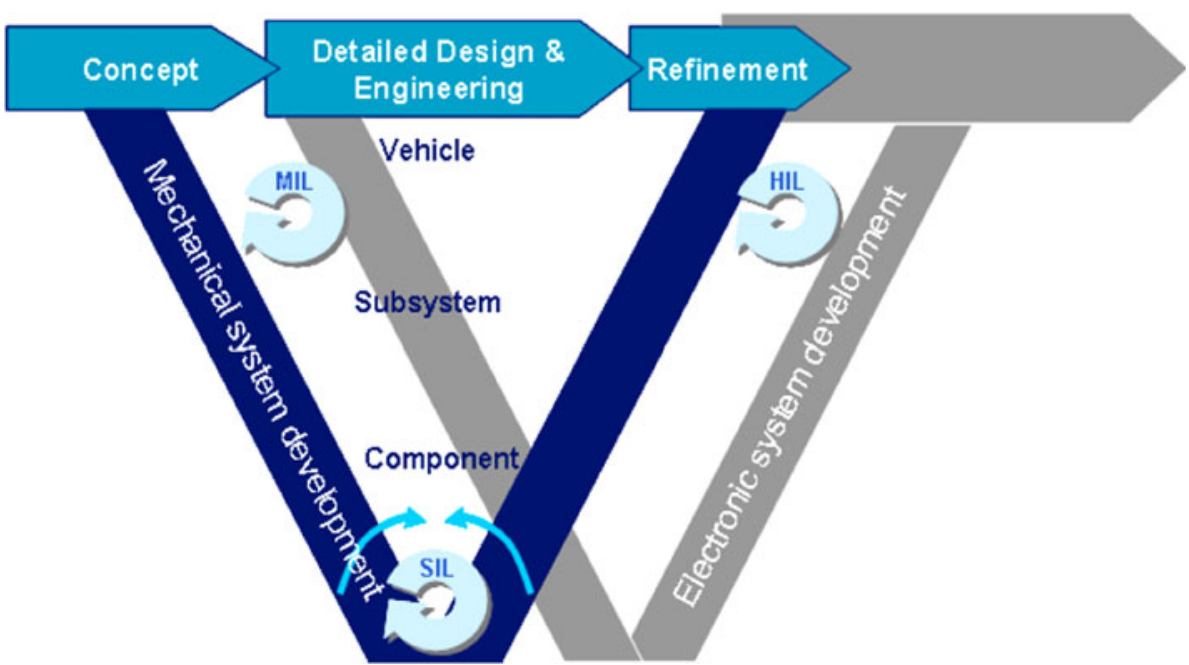

models must have an interface to vehicle networks such as the CAN (controller area network) bus, where the status on the control systems and sensor signals is available.

Both control systems development and mechanical systems development adopt the so-called "V-approach" [3, 4, 19-21], propagating system-level requirements to component design and validating the system performance at increasing integration levels. As Fig. 1 illustrates, the engineering of mechatronic systems requires the application of two interconnected "V-cycles": one focusing on the multi-physics system engineering (like the mechanical and electrical components of an electrically powered steering system, including sensors and actuators); and the other focused on the controls engineering, the control logic, the software and realization of the control hardware and embedded software.

Figure 2 shows for the example of engine design how models of various abstractions are used at the various design levels.

This approach includes the use of OD requirement models, 1D functional and physics models down to 3D detailed design models. The terminology $0 \mathrm{D}, 1 \mathrm{D}$ and $3 \mathrm{D}$ is simulation jargon. The term 3D model refers to the fact that the model is clearly associated with a geometry. For example in finite element models, the mesh is directly linked to a geometry. In 1D models, this connection is lost. Components, systems or subsystems are represented by icons to which mathematical equations are connected. OD models describe systems, not with mathematical formulas, but with simple relations or tabular representations. Testing takes place first at the level of components and then at various levels of integration, while full vehicle tests provide the validation in integrated and operational conditions. The final implementation of the control software on the embedded electronic circuits is tested using hardware-inthe-loop (HiL) test benches. The latter term is further explained in the text.
Up to present, this process is however very little integrated, with a clearly separated mechanical and electronic/ control design cycle and hence failing to address the need for integrated and maximally frontloaded system modelling. Many integration problems become obvious only at a very late stage of the design and are hence very costly to resolve. The challenge in this process is to enable a mechatronic system engineering approach that can be used throughout the complete design process, based on scalable and interoperable simulations, including their application to target setting, concept system engineering, functional simulation, 3D detailed simulation and test validation. Some further comments on the various challenges follow below.

\subsection{Multi-physics system modelling, simulation and validation}

To engineer intelligent systems, an expanded need exists for multi-physics system modelling, simulation and validation. For example, the performance engineering of an electrically assisted steering system requires a combination of mechanical and electrical system modelling. A brake system requires mechanic, hydraulic and electric system models. An engine requires models for combustion, kinematics, dynamics and structural analysis, including specialized models for bearings.

Multi-physics system modelling, simulation and validation also need to handle an increasing diversity and complexity of sensors and actuators that are used in mechatronic systems and to take into account the environment in which the system will operate. For example, to simulate the working of an active cruise control in a vehicle, one needs the modelling of driving scenarios including traffic (like approaching vehicles), the modelling of the functioning of the radar that is used as sensor for traffic and the integration with vehicle dynamics. When 
Fig. $2 \mathrm{~V}$-process for an engine system

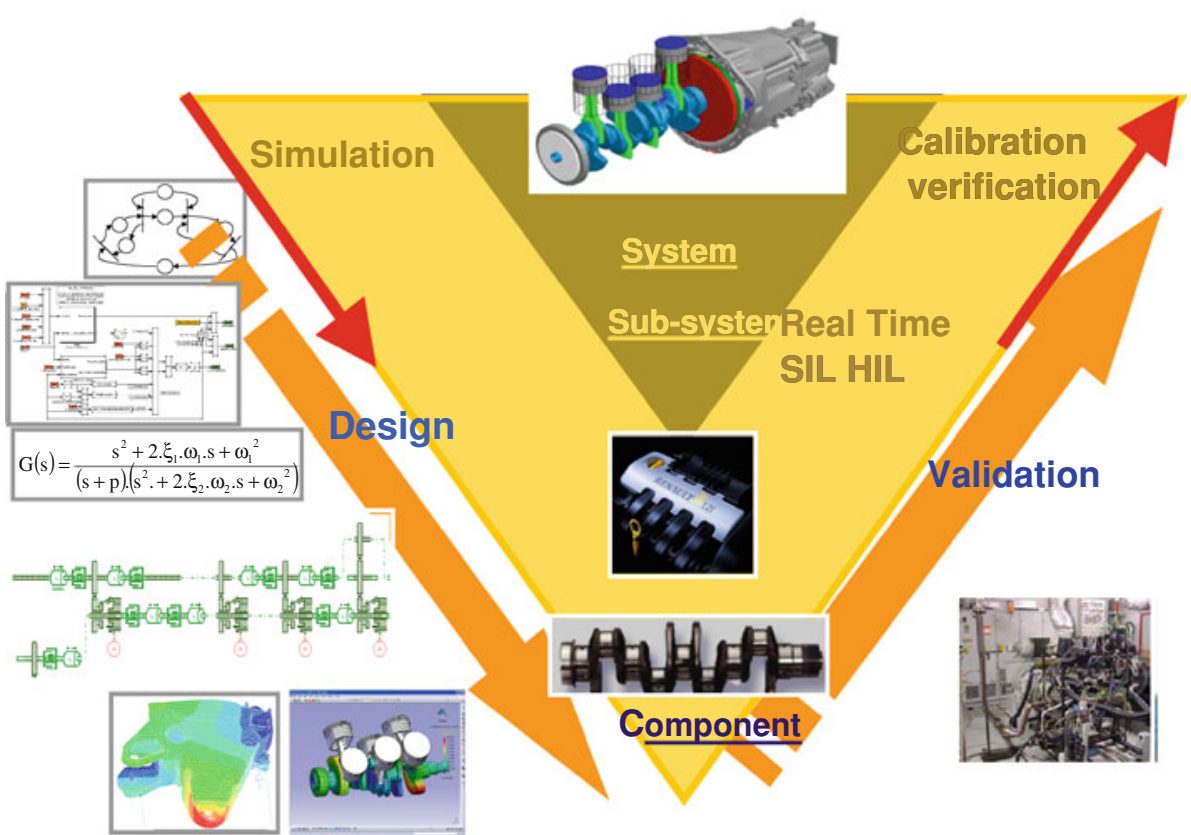

additionally combined with vision systems (cameras), one needs the simulation of driving scenarios in a virtual environment with high realism, to simulate the functioning of the vision system for operation in different weather (rain, fog...) or light conditions (day, night...), to properly validate the functioning of the vision system and how it will interact with the vehicle dynamics.

Integrating such models of different nature is always a challenge. Interoperability requires common frameworks for variables and functions and well-described interfaces. The most challenging element to a realistic system-level performance description, based on heterogeneous models, is to link the world of 1D system and functional simulation with 3D geometry-based simulation (e.g. multi-body or structural/vibro-acoustic FEA/BEM models).

When the 3D structural model is a time-domain model, for example a multi-body simulation (MBS) model, the 1D and 3D models can be both expressed in terms of state equations which are solved through time integration. MBS models are typically used to calculate connection forces and macroscopic displacements of rigid, connected, systems such as mechanisms, drivelines, brake and suspension components. The basic model parameters are the inertia and connection properties. The 1D models are then used to describe the electric or hydraulic actuation, simplified models for combustion, tyres, external loads, etc.

The actual model integration and calculation can then be executed in co-simulation (see further), or the system equations of one model can be embedded in those of the other model [22-25]. This situation is characteristic for applications such as vehicle dynamics, internal engine dynamics, aircraft control surfaces, satellite antennas, etc. An example of such a model is discussed in Sect. 4 for the case of an active vehicle suspension.

The 1D to 3D model coupling problem is more complex for the case of the classical 3D frequency-domain simulation approaches for the structural and vibro-acoustic behaviour, and which are based on the use of finite element analysis and boundary element models (FEA, BEM). Such models are used to calculate the internal stresses and displacements/velocities inside and at the surface of components. They are the basis for noise, durability and structural dynamics studies. These methods are, however, not directly compatible with the time-domain approaches needed to model, simulate and optimize control system performance. In general, the structural model is furthermore too large to be directly transformed into an equivalent state-space model and to serve as basis of controller design or in time/ frequency response analyses for checking the controller performance. Still, the connection to multi-physics 1D models can be needed for examples such as noise control, or to control the dynamics of flexible bodies. Essentially, two approaches can be distinguished to realize this:

- Reduction of the structural model to an equivalent loworder state-space model. This is the standard approach to couple structural and control models. Several model reduction methods are available such as modal reduction, Krylov reduction, SVD reduction and others [2630]. An application of the use of reduced models to control design is in the active control of sound and vibration where reduced structural models are included 
Fig. 3 Integrated mechatronic simulation approach
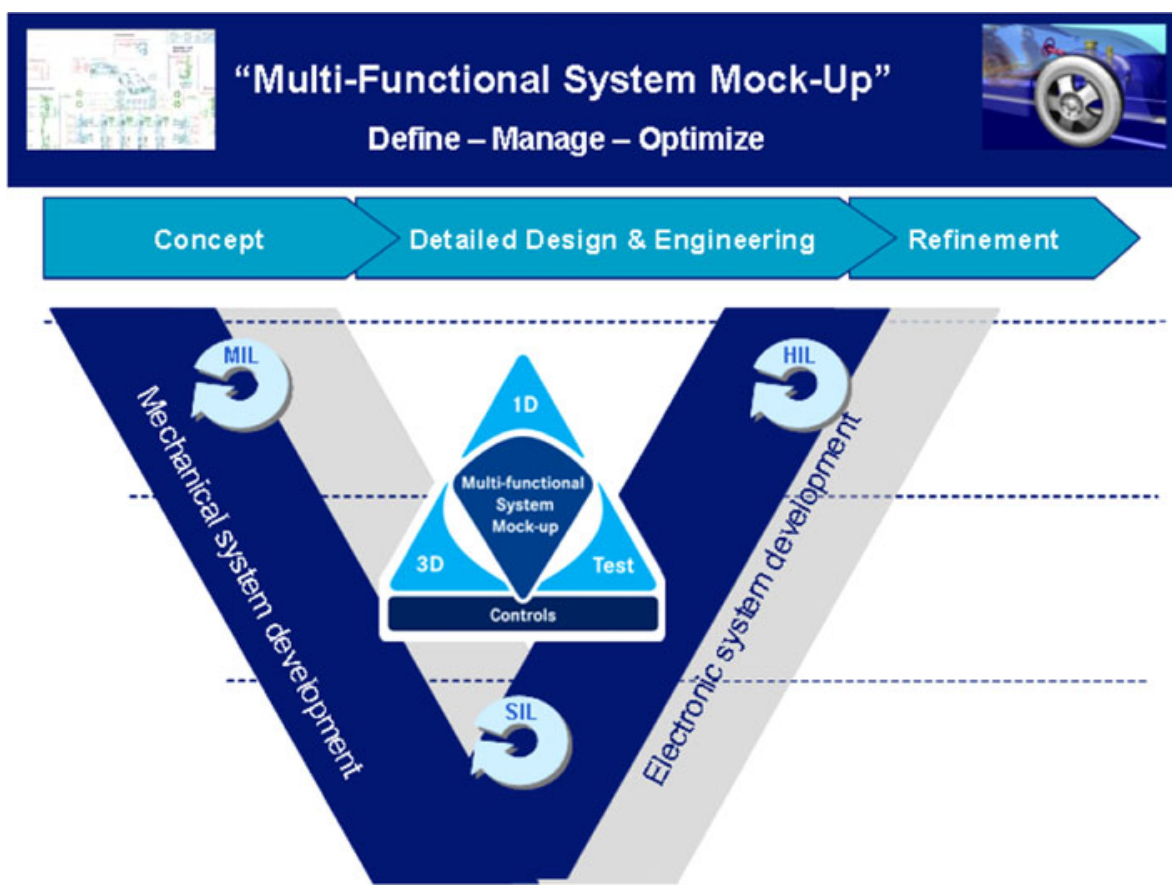

as state-space models in a time-domain model of the controlled system. The model reduction factors can be very high, reducing a full structural model to sizes of typically 20 to maximally a few hundred states. An example of such application is discussed in Sect. 5 for the case of active noise control.

- Integration of the 1D system simulation concepts inside the FE model formulation. This approach keeps the original complexity of the FE model, but adds for example actuator/sensor and control elements by additional degrees of freedom and constraints and the use of special circuit elements [31]. Changes in these elements can then be separated out from the global system behaviour through the use of a superelements approach for the non-varying part. Examples are typically found in smart materials applications where the focus remains on the material and geometric aspects and some form of idealized control is used [31].

It is important to stress that mechanical and electrical/ electronic system models must be integrated as soon as possible in the design process, enabling to reduce or even eliminate the divide between the $2 \mathrm{~V}$-cycles of Fig. 1 and leading to an integrated, multi-functional system mock-up approach to build the mechatronic simulation model (Fig. 3).

\subsection{Connecting multi-physics system engineering to controls engineering}

The second key challenge is to integrate the system and the control models. As shown in Figs. 1, 3 and 5, the objective is to achieve this in all stages of the design process, such that the V-cycles of multi-physics design, control and embedded software design get really integrated. In the figures, multi-physics design is denoted by mechanical system development because of the original mechanical nature of vehicles. Control and embedded software design is referred to as electronics system development.

All these stages of control engineering require interaction with multi-physics system engineering. One can distinguish the following phases:

1. The combination of the multi-physics simulation model with that of the controller, to enable the design of the control logic and the performance engineering of the intelligent system. This is referred to as "model-in-theloop" (MIL). The simulation is "off-line", i.e. there is no requirement for real-time performance of the simulation.

Basically, two interconnection objectives can be distinguished: one is to perform systems engineering based on the multi-physics "plant" model, including the application (and hence representation) of control (Fig. 4a); the other is to perform control engineering, including the model of the systems "plant" model (Fig. 4b).

The first objective for example serves the purpose of configuration design (how many actuators, where to place them...) or concept evaluation studies or the optimization of the mechanical system design taking into account the presence of control and certain control laws (or even systems).

The second objective is oriented to the development of the optimal control logic, the development and verification of control hardware, control libraries and embedded 


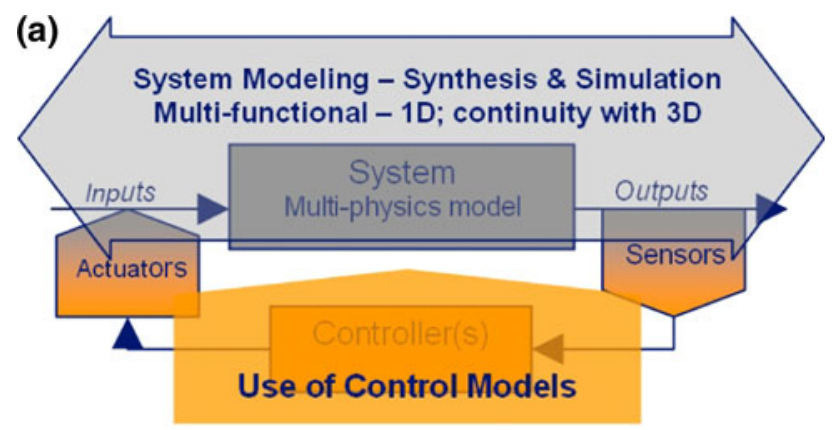

(b)

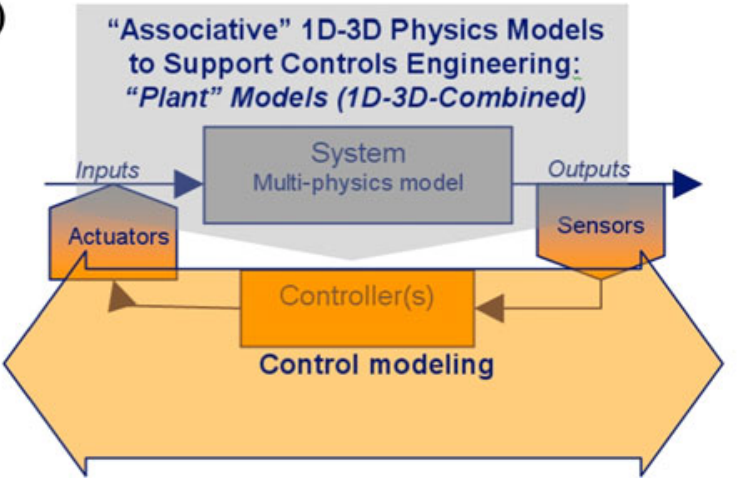

Fig. 4 a Systems engineering with control models. b Control engineering with systems models

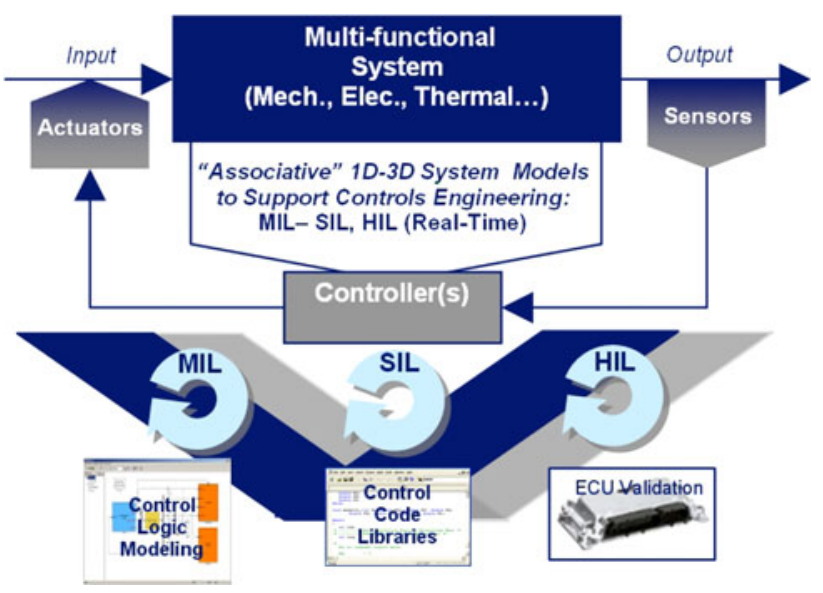

Fig. 5 Associative 1D-3D models

software up to the validation and calibration of the control system on the electronic control unit or ECU (Fig. 5).

To couple the models, different approaches exist. One may embed state equations with a description of the plant system (e.g. MBS or 1D model) into these of the control (or vice versa) to enable the use of one solver, or adopt a true co-simulation approach where each system part runs its own solver [22-25]. Figure 6 shows a summary of various approaches for the case of an MBS and a CACE (computer aided control engineering) model.
Alternatively, or in combination with the above approaches, a reduction of the plant model (e.g. an FE or complex, even non-linear MBS model) into a description compatible with the controller model (e.g. state-space formulation) may be required. Model reduction is a wellestablished field, covering a large spectrum of techniques, as was discussed for the problem of multi-physics simulation. The approach for controls development is just one specific case, the 1D model being a control model. The model reduction step mostly achieves its goals at the expense of the full observability and/or controllability of the physical phenomena, leading to a macroscopic 'equivalence', but losing direct insight into the microscopic observation domain. The challenge is to develop model compression methodologies that allow maintaining a relation with the physical meaning of model parameters.

Such co-simulation and model reduction approaches are used both for MIL applications for systems engineering and for control logic engineering.

2. The next step is the development and optimization of the "embedded" control software. This needs also to be done in context of the functioning of the multi-physics system to be controlled. This is referred to as "software-inthe-loop" (SIL). Whereas some of this can be done in offline simulation (provided software libraries of the controller are available), the final optimization needs to take into account the working of the software in real time, requiring real-time capable multi-physics simulation models.

3. The final testing and calibration of the controller software and hardware require the controller to be connected to a multi-physics simulation model of the components, subsystems or system, in a dedicated computing environment that is referred to as "hardware-in-the-loop" (HIL) [32]; of course, this requires real-time capable simulation models.

From the SIL and HIL problem definition, it is obvious that one of the critical problems in running multi-physics models in a control context is their affordability in real time, requiring to identify the best trade-off between model compression and real-time affordability. It also requires deriving criteria for assessing accuracy and reliability of real-time models in a run-time environment.

Of particular importance to the in-vehicle application is to map the process of real-time systems and embedded software to the AUTOSAR standard. AUTOSAR (AUTomotive Open System ARchitecture) is an open and standardized automotive software architecture [33], jointly developed by automobile manufacturers, suppliers and tool developers. Defining a platform-independent development approach is key to make best profit of technology development. Hardware platforms for embedded system modelling and development must hence comply with industry 


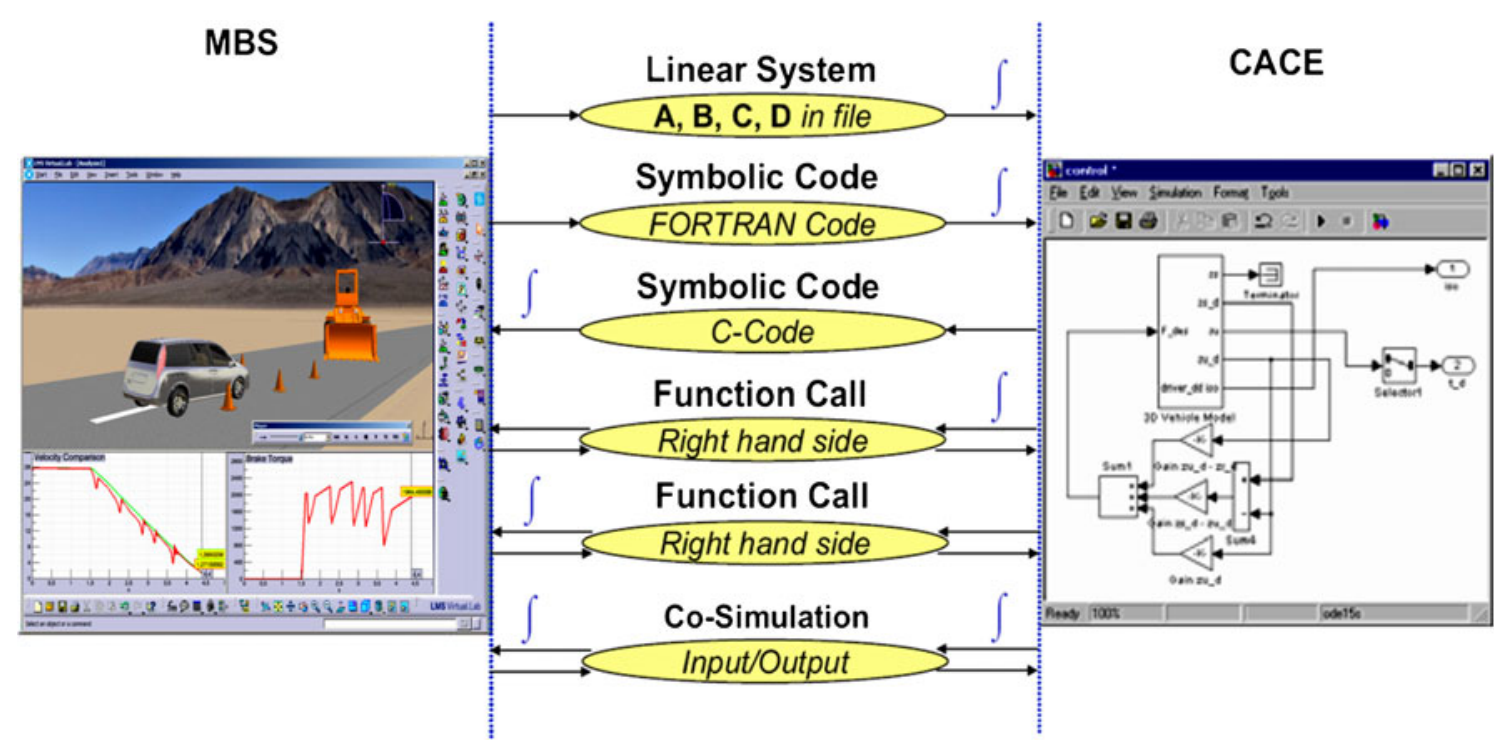

Fig. 6 Co-simulation approaches

standards such as AUTOSAR, which is gaining large consensus. Also for testing intelligent systems, new challenges emerge, by providing testing systems that have the most complete interfacing to vehicle networks and standards (CAN now, but tomorrow FlexRAY and others), so as to enable best measurement and analysis of a product performance in the context of the operation of the controller(s).

To illustrate the practical deployment of the discussed mechatronic simulation approach, a number of industrially relevant design cases are discussed addressing one or more of the presented elements of this approach. One case focuses on time-domain co-simulation, and the second one on integrating frequency and time-domain modelling.

\section{Case: application to vehicle dynamics}

The discussed concepts of multi-physics simulation and system-control modelling integration have been applied in the design of advanced vehicle dynamics solutions. Such design engineering task comprises multiple phases, each requiring specific modelling and simulation actions. First, the global vehicle dynamics performance was modelled in view of the assessment of the performance gain of a vehicle dynamics control solution. This essentially required the cosimulation of the 3D vehicle driving dynamics model and an idealized control system. Secondly, the active suspension was designed in detail. This required identification of the optimal control gains using a 1D-control co-simulation approach and the design of a dedicated active damper using a multi-physics actuator model. Finally, a test rig for testing shock absorbers was developed allowing the validation and optimization of the stand-alone damper hardware using system models for representing the vehicle integration. This "hardware-in-the-loop" approach allows the early testing of new actuator designs taking into account various potential vehicle integration models; hence, without that the actual prototype vehicle needs to be available.

\subsection{Vehicle-level system and control co-simulation} approach

This first presented study demonstrates the application of the system and control simulation integration approach to the problem of building models for vehicle ABS (anti-lock braking system) evaluation.

Multi-attribute models are developed to optimize and balance vehicle performances such as handling and road noise. The typically optimized parameters are hardpoint locations as well as suspension bushing stiffness values [34-36]. The key model used as the basis for this is a multibody simulation (MBS) model made in LMS Virtual.Lab Motion. The MBS model allows optimizing the driving performance, taking into account expected driver behaviour. From this model, loads can be derived for use in body and chassis durability calculations and predicting acoustic performances. The MBS model was then used in a cosimulation approach with MATLAB-Simulink to develop control algorithms for active safety purposes [37]. Benefit has been made of the capability of each package to use its own integration algorithms tuned for the typical problems facing it. 
This also allows implementing and tuning the control algorithms without losing the complexity of the complete virtual prototype. Figures 7 and 8 show part of the MBS model (front axle), the ABS control model and some simulation results.

A VDC (vehicle dynamics control) system is used to control the lateral dynamics of the vehicle, especially in scenarios with larger lateral acceleration and lower road friction. One typical way of control in VDC is to use ABS to generate braking torque, independently for the four wheels according to the inputs of sensors for steering wheel angle, yaw rate, lateral accelerations, etc., and the drivers' command regarding desired vehicle behaviour. Figure 9 shows the VDC data flow within the closed vehicle systemcontrol loop.

Two ISO standard manoeuvres were adopted during the simulations, Step Steer (ISO 7401) and Double Lane Change (ISO 3881), with VDC on and off. Figure 10 shows the resulting path of the vehicle with/without VDC under a step steer manoeuvre, clearly showing the difference between the lateral displacement responses.

Figure 11 shows the results of the yaw rate of the vehicle with/without VDC system. The obvious improvement in yaw rate response shows the effect of VDC on lateral dynamics of the vehicle. In particular, a greater effect is observed after a certain period of time when the
VDC system suppresses effectively the transient yaw vibration due to the impulse at the steering wheel.

This example shows how the use of multiple models can be used to simulate the complex performances of the chassis system and to evaluate and optimize the settings of the integrated VDC control system.

\subsection{Mechatronic optimization of an active damper}

The second study related to the engineering of an advanced vehicle dynamics solution describes the use of a multi-level simulation approach to the design of an individual component of an active suspension system. A $1 \mathrm{D}$ simulation model integrating vehicle behaviour and control is used to derive system loads. These loads are then used with a detailed component model in an optimization loop to derive design parameters for the component.

The design of an active suspension is more than the design of a control law. The control law will steer actuators that interact with the chassis of the vehicle and the measured response of the vehicle will affect the new computed values of the controller. Therefore in order to optimize the active damper, the chassis dynamics and the control law need to be taken into account [38, 39].

The active damper, manufactured by Tenneco, is a hydraulic type, consisting of a hydraulic single rod
Fig. 7 ABS-chassis system co-simulation model
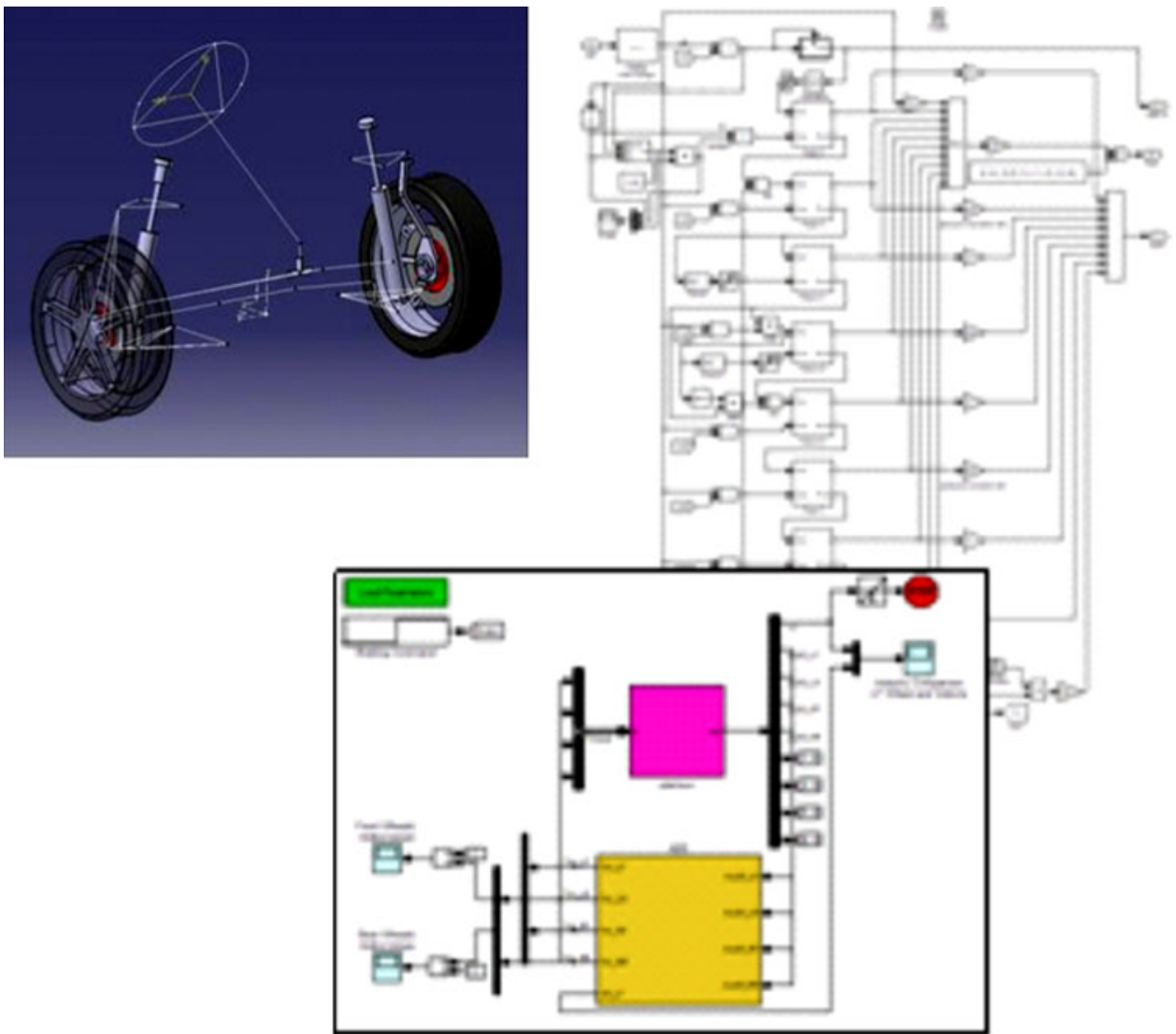
Fig. 8 ABS-chassis co-simulation results
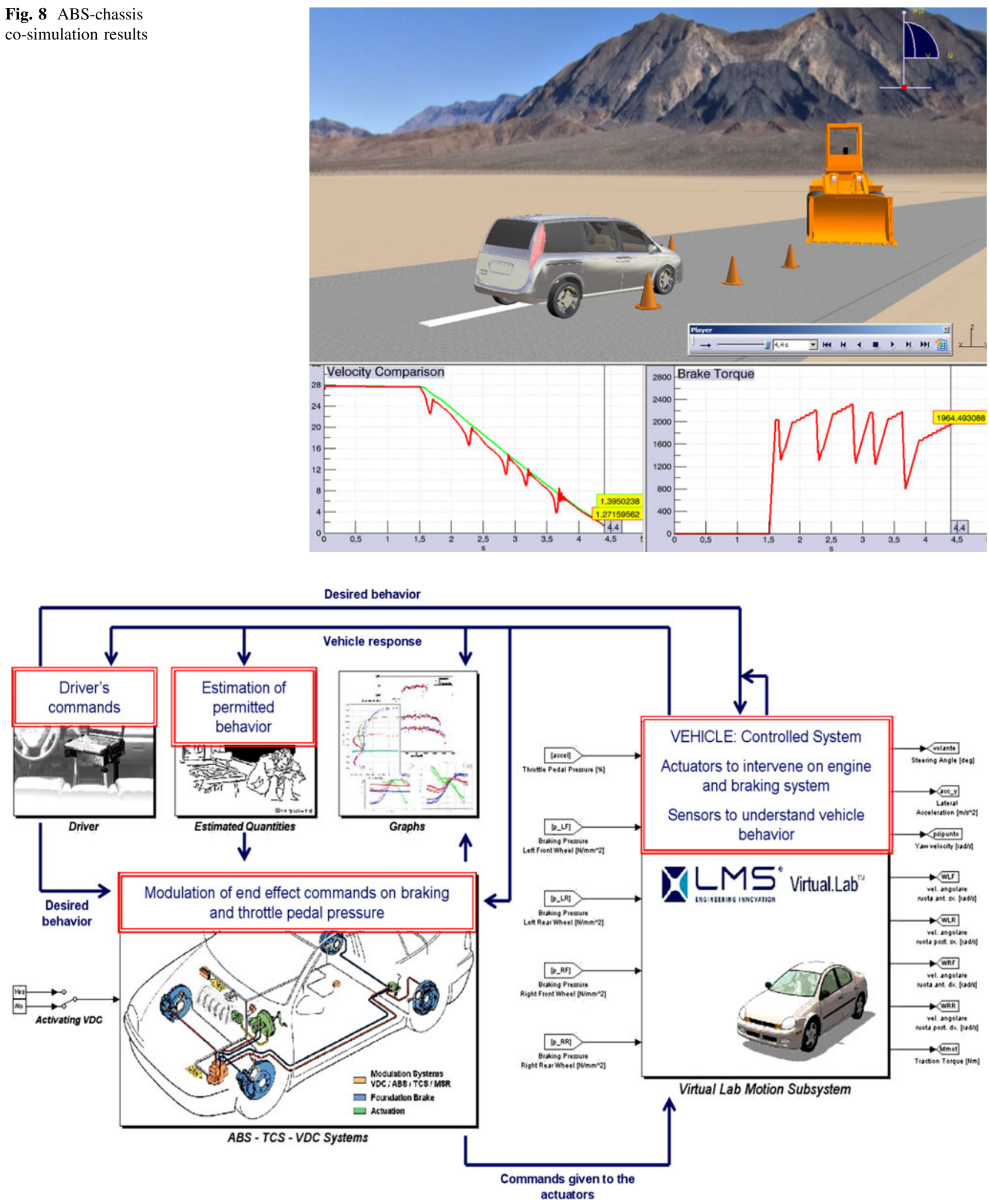

Fig. 9 VDC model and data flow 
Fig. 10 Steep steer trajectory (VDC on/off)

Fig. 11 Yaw rate (VDC on/off)

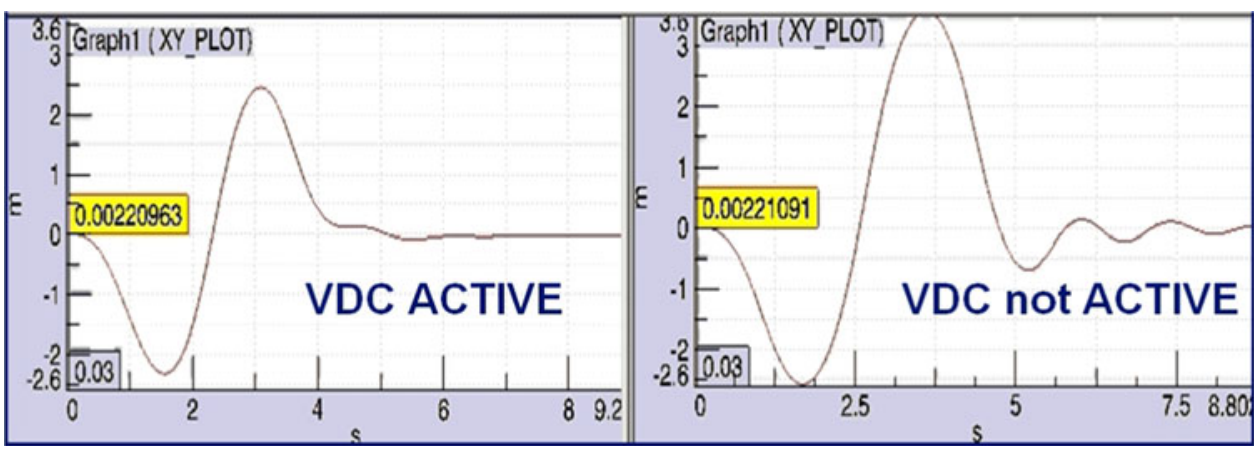

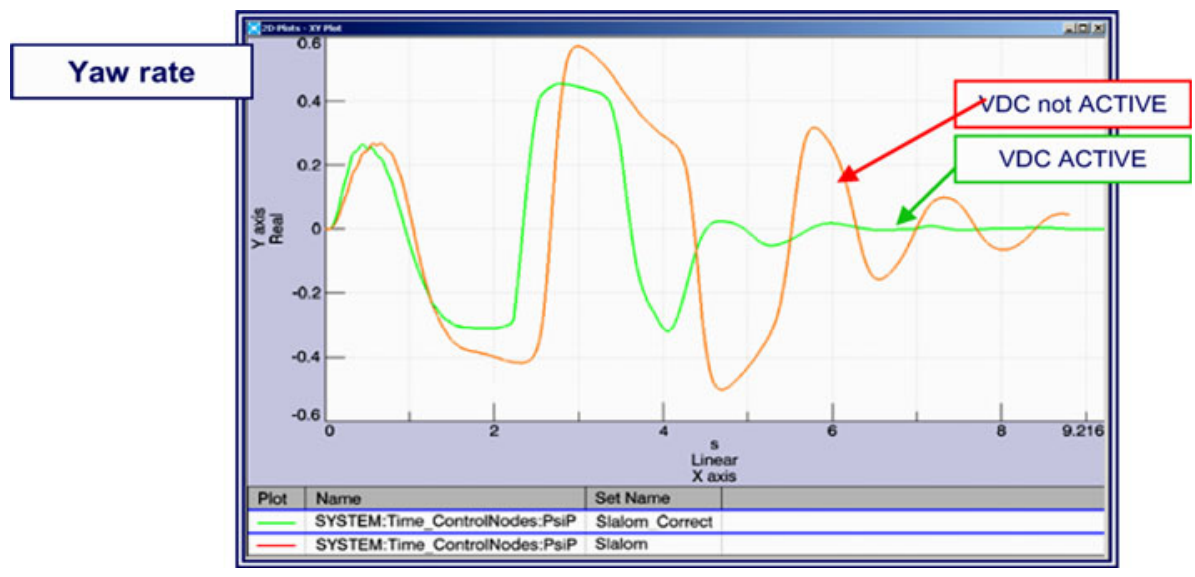

cylinder, two valves and a pump [40]. The objective is to optimize the cylinder and rod diameters, the pump flow and the characteristics of the valves with respect to energy consumption while meeting some comfort and ride and handling performance criteria.

The optimization is performed in two stages (Fig. 12). Theoretically, and regardless of physical feasibility, every desired performance can be achieved provided that sufficient energy is pumped into the system. Therefore, in a first stage, the set of active dampers is determined that can meet the desired performance. In the second stage, the damper parameters that deliver the lowest power consumption are selected from the set of the first stage. In this way, the damper is obtained with lowest power consumption while meeting the performance criteria with respect to comfort and ride and handling.

The split in the two stages is possible because of the control structure designed by Tenneco. The controller consists of a master controller which is a kind of sky-hook control algorithm that processes inputs from accelerometer, suspension deflection, steering, throttle and braking data into the desired forces that need to be applied by the active dampers to the four corners of the car (approach similar to [41]). Distributed control, the so-called actuator management, ensures that the requested forces are generated by the active shock absorber.
A behaviour model, implemented in LMS Imagine.Lab, is used in the first stage of the optimization (Fig. 13).

It consists of a 15-degree-of-freedom car model, power train and braking system, front and rear suspension including the elasto-kinematics and tyres (Pacejka model). Instead of implementing the active shock absorbers in the model, the forces computed by the controller are immediately fed into the suspension. In this way, perfect actuator behaviour is assumed. The master controller is tuned such that the desired performance is met. Once the master controller is tuned, force-velocity couples are calculated to determine the set of damper parameters that can realize the required performance.

The second stage consists of a detailed model of the damper, built with the hydraulic component design library in LMS Imagine.Lab (Fig. 14). Using force-velocity couples from the most occurring road profiles, the optimal damper parameters with respect to energy consumption are selected. The optimization is a mixed integer problem, involving a discrete parameter set, solved with Optimus. Three valve families are available. Within a family, the course of the valve characteristics is similar. Therefore, each valve can be represented by a basic characteristic and some scaling parameter. The optimization delivers the piston and rod diameter, the maximum pump flow and the selected valve characteristics. 


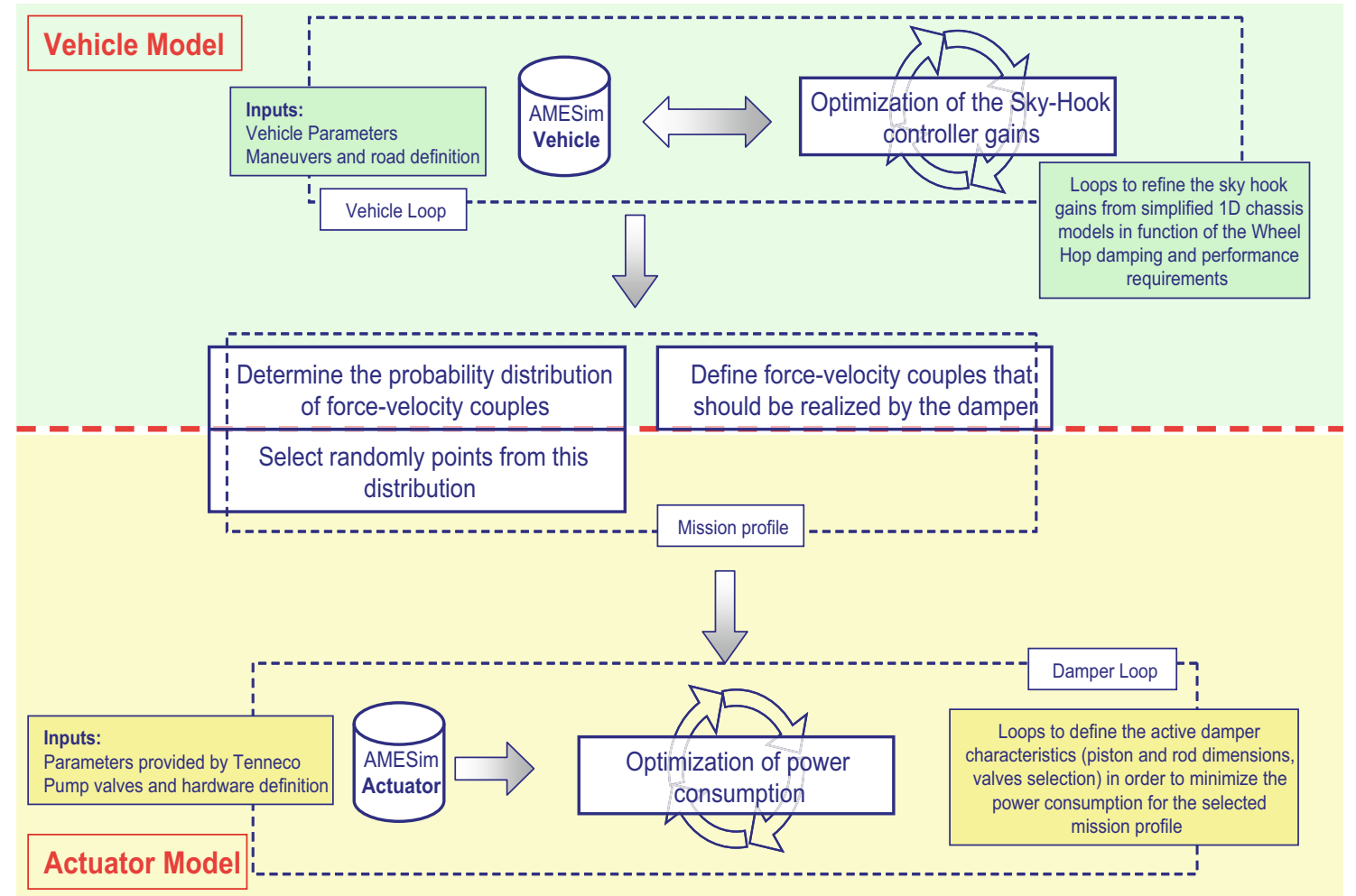

Fig. 12 Active damper simulation approach

Fig. 13 Active suspension 1D model

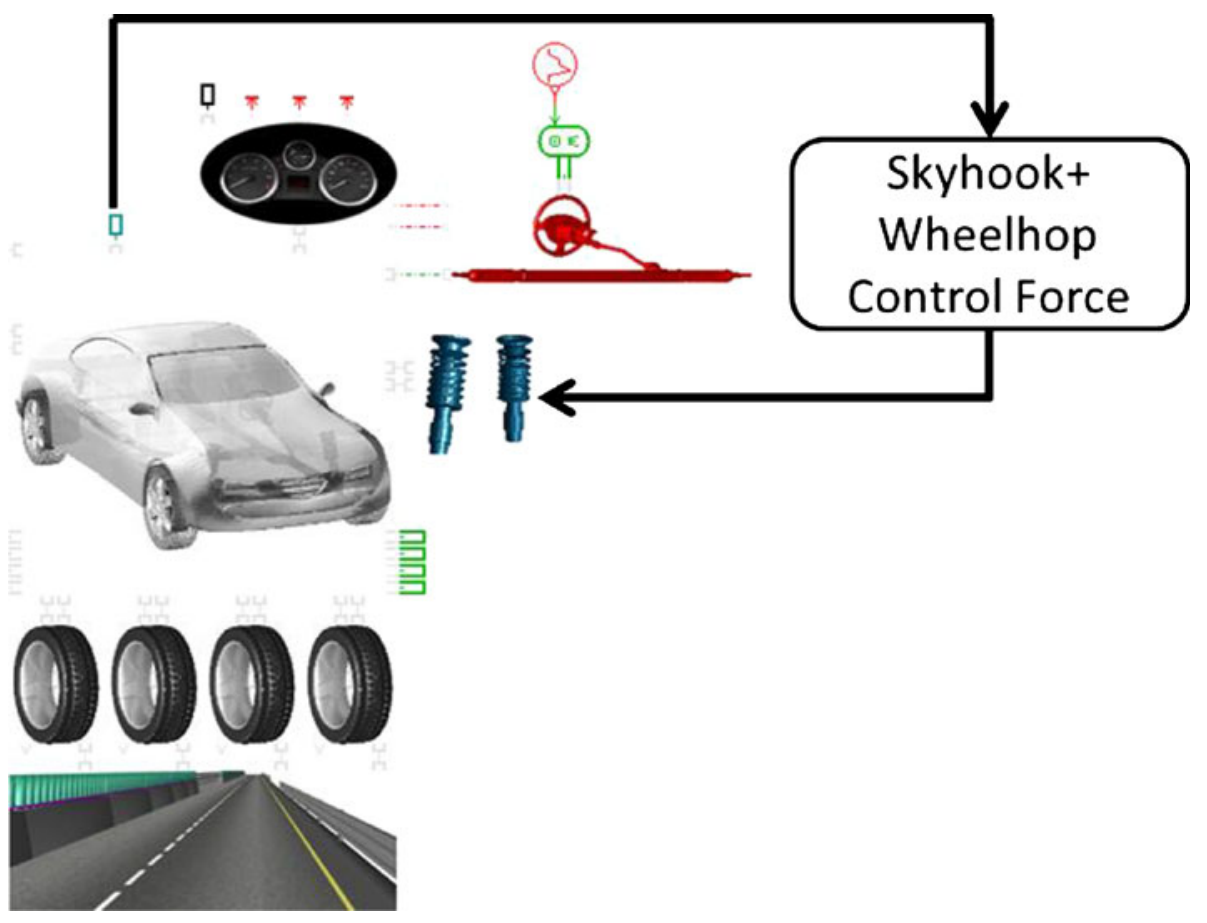



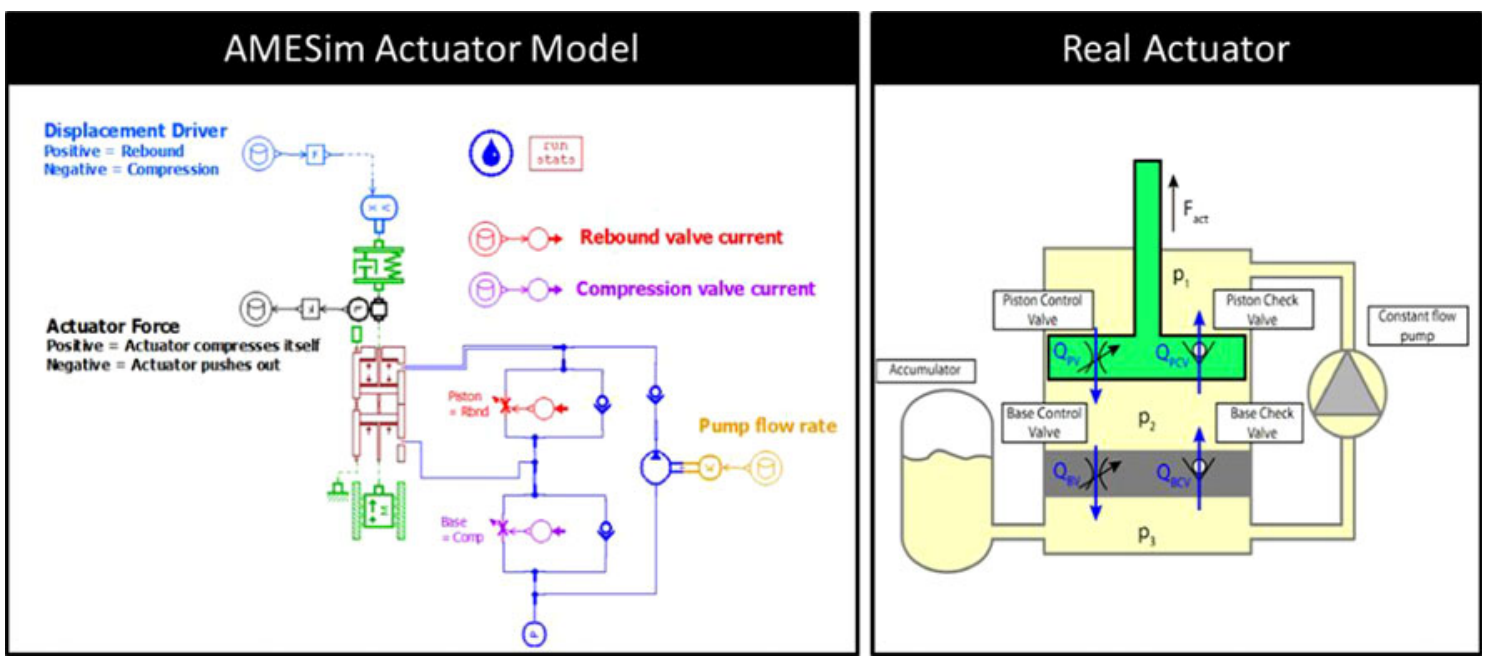

Fig. 14 Damper 1D model (left) and simplified Z-translation model (right)

After the optimization, a power consumption reduction of $50 \%$ was realized with respect to the initial configuration.

\subsection{HIL testing of a passive damper}

A car is a complex system of individual components that interact with each other. Therefore, the performance of the component should be assessed within the environment it is placed in. This can be performed within a complete virtual environment or in a prototype. In the context of upfront engineering, an evaluation in a combined virtual environment with physical components, the so-called hardware-inthe-loop (HIL) can be beneficial. A typical scenario can be a supplier who must validate the performance of a component in a car while no car prototype is available yet. In the present work, the component to be tested is a passive shock absorber.

The HIL process consists of several stages. First, a model of the environment of the component needs to be synthesized. In this case, this is the car, from which one shock absorber is removed. The car model is implemented in LMS Imagine.Lab and consists of a 15-degree-of-freedom chassis equipped with front and rear suspension. Pacejka tyre models, simplified braking and power train system are included (Fig. 13). Steering angles and road profiles can be specified by the user. This model is converted to a real-time environment and run using the realtime solver.

As the model needs to interact with the physical world, the timing of in- and outputs should correspond to the real world. At the sample interval, the model and the component in the physical world exchange information. This means that the simulation of the model for the next sample interval should be completed within this interval. To achieve this, a deterministic solver is selected, in this case a fixed step-size solver. A critical issue is selecting the time step, such that the model converges and the required accuracy of the variables of interest is achieved.

This selection is also related to the specific hardware platform (computer or DSP) on which the real-time model will run. In case the timing cannot be achieved, model simplifications must be made. For this project, the elasto-kinematics of the vehicle have been removed and the sample interval was set to $1.2 \mathrm{~ms}$. The hardware to run the model is a Pentium M $1.4 \mathrm{GHz}$ with $1 \mathrm{G}$ Byte cash in PC/104 plus format from Kontron. The model is run in a Debian Linux environment patched by RTAI to make it real time.

To transmit the computed loads from the model to the damper, an electro-dynamic actuator is applied. To realize the requested loads, an actuator control system needs to be designed. An important issue is the selection of the output variable of the model. In case of the damper, this can be the displacement/velocity or the force. It is generally known that a displacement loop has a lower bandwidth than a force control loop, but on the other hand, a position control loop is much more robust and easy to stabilize than a force control loop. In this case, a position control loop is hence selected, providing good signal-following characteristics up to $10 \mathrm{~Hz}$.

Finally, the response of the system, the force, needs to be measured and fed into the model. This creates a closed loop system. Because of non-ideal behaviour of the actuator, instabilities may occur. Therefore, an additional stabilizing control loop is installed. Figure 15 shows an overview of the HIL test setup.

Based on the choices made, the HIL system is able to validate the shock absorber up to $10 \mathrm{~Hz}$. It is clear that 


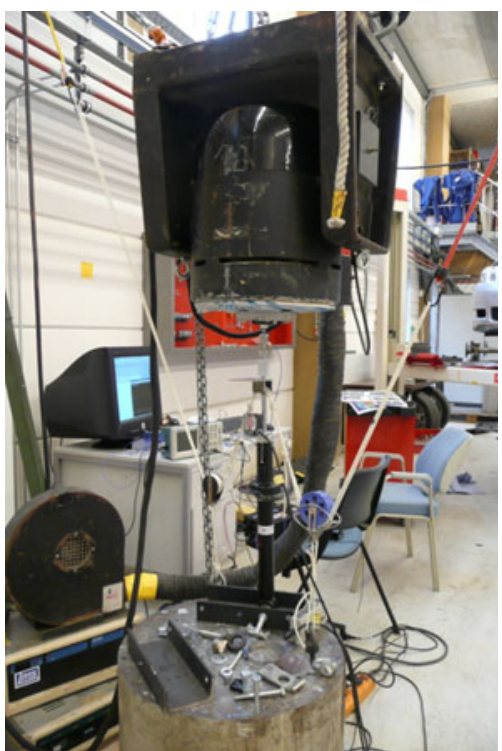

Fig. 15 Damper HIL test setup

during the design of an HIL, several decisions need to be made that determine the final performance.

\section{Case: active noise control}

In a second case study, the application of a model-based system engineering approach to active noise control is discussed. Model reduction into a multi-physics timedomain simulation representation is the key to the mechatronic simulation approach for this case.

Active noise reduction (and sound shaping) is a widely studied research topic with many potential industrial applications. Next to purely acoustic control, a structuralacoustic control approach is increasingly adopted. Multifunctional or active materials can be used as sensor and/or actuators, which, when coupled to a control system, form intelligent structures. These structures allow reducing costs and required space and the number of elements in the system. Most such systems are, however, developed as stand-alone "add-ons" without considering their effect as part of the global controlled system in the design. A modelbased integrated mechatronic engineering approach may hence bring significant added value.

The modelling problem basically consists of relating the large-size 3D, frequency-domain (FE, BE-based) vibroacoustic and structural models for the vehicle structure and structural components, interior vehicle cavities and exterior propagation field, with models of smart material sensors and actuators and a time-domain control model (Fig. 16).

The main approach hereto is model reduction, allowing incorporating the reduced model as a plant model in the controller simulation. Very large reduction factors are typically used, reducing the large FE models to timedomain 1D (typically state-space) models of realistic size (20-200 degrees of freedom).

Sensors and actuators are often represented by 1D models for their functional performance, while their added mass and stiffness are accounted in the 3D FE models. The acoustic propagation can be related to the structural outputs by means of an acoustic transfer vector approach.

This approach was applied to the active firewall control of a vehicle-like test setup with piezo-patches to reduce the interior noise [42, 43].

The modelling procedure to derive the state-space model starts with the structural FE model and features the possibility of incorporating sensors and actuators models to the FE/FE vibro-acoustic model. It includes the following steps using multiple software tools (Fig. 17):

- Generate structural mesh and apply material properties (FE pre-processor)

- Add actuator and sensor mechanical models (FE preprocessor)

- Run a modal analysis (FEA)

- Build the acoustic FE model of the engine cavity (EC) and passenger cavity (PC) and perform modal analysis (FEA)

- Import the structural model and couple it with the acoustic one (FEA)

- Calculate actuator and sensor electro-mechanical coupling (extended FEA)

- Reduce and convert the FE model into a state-space model (Matlab)

- Implement and optimize the controller with the coupled state-space model (Matlab/Simulink).

The coupling between acoustical and structural models is shown in Fig. 18. After performing a coupled modal analysis, the desired degrees of freedom (DoFs) are taken to derive the state-space (SS) model. In this case, the SS model features two inputs ( 1 actuator on the firewall and a sound source in the EC) and four outputs ( 3 pressures in the PC and one velocity on the firewall). The SS model derived from this coupled approach allows the implementation of any controller involving the pre-defined DoFs, and if the FE approach involves the systematic representation of the sensors and actuators, the resultant SS model is, in fact, a representation of the fully coupled electro-vibro-acoustic system, with any possible input/output relationships allowed by the chosen DoFs.

Using this model, an optimization procedure is performed using OPTIMUS as simulation management and optimal search engine. OPTIMUS is able to manage the structural analysis made by MSC. Nastran, the acoustic analysis for a coupled vibro-acoustic model made by Virtual.Lab Acoustics and, finally, a controller simulation 




Fig. 16 Active noise control system approach
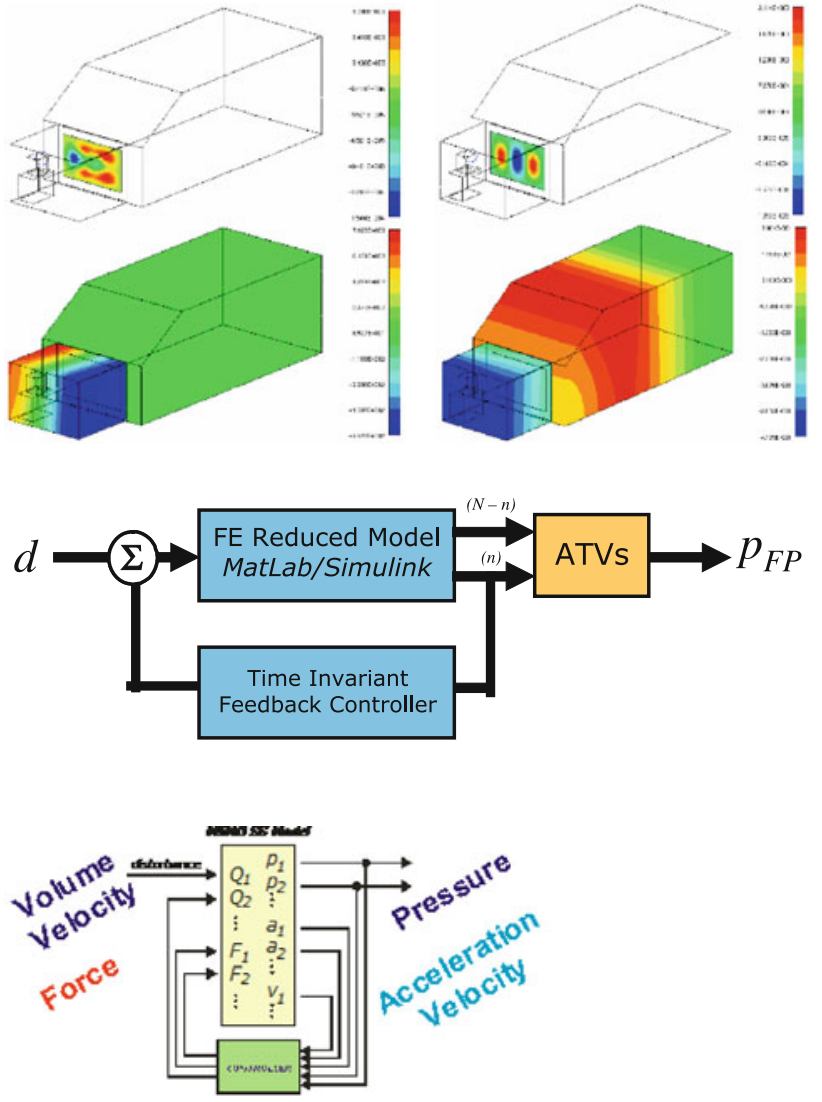

Fig. 17 Active firewall mechatronics model: 3D structural model (upper), reduction as plant model in the control model representation (middle) and final state-space model (lower) using a state-space model. A crucial point is efficient provision of reduced models for different parameter settings.

The cost function takes into account three parameters: the sound pressure level at the drivers' head (performance), the input energy from the actuator (effort) and a penalty for the total mass (weight) of the structure, representing the financial cost impact. The variables are the firewall thickness and the gain of the velocity feedback controller. Initially, the position of the collocated sensor/actuator pair (SAP) is considered fixed, based on previous analysis; in a further step, this parameter is also included in the optimization loop.

Figure 18 shows the cost function for each thickness in function of the feedback gain, on the best SAP position for each case. There is an optimum gain for each thickness and SAP position. It is obvious that the best SAP position and optimal feedback gain depend on the thickness, which indicates that the global optimum can only be achieved in such a concurrent design. This demonstrates the validity of using an integrated mechatronics simulation approach.

A more extensive discussion of the various modelling aspects and the detailed optimization procedures can be found in [42-46].

\section{Discussion and future research}

What becomes clear from the above discussion is that, on one hand, the use of system models and simulation is an essential element in the product design and engineering 
Fig. 18 Cost function for each thickness and best SAP position

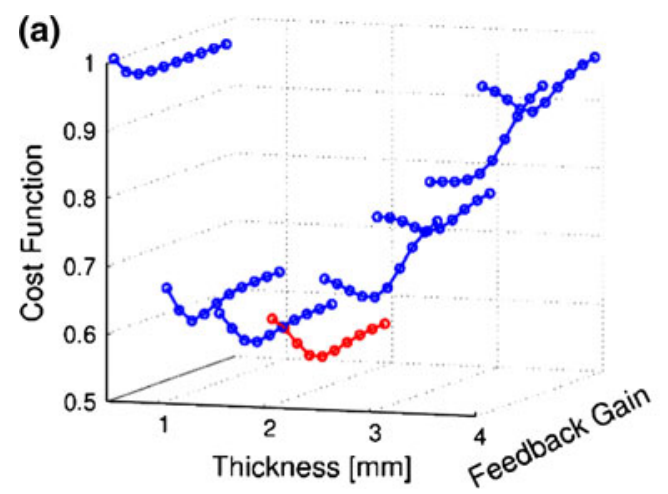

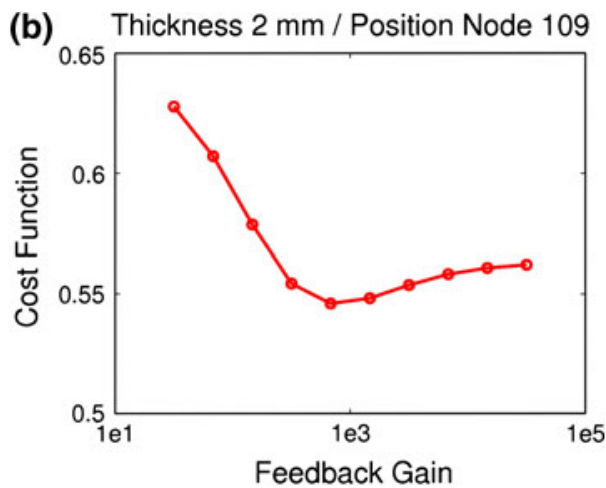

process, but that on the other hand, there does not exist a single uniform simulation methodology that covers the modelling requirements of the complex systems as used in today's products. Different product functions and performances require different models, simulation methods and tools. Modelling is pervasive throughout the whole product design process, from requirement analysis to component design and again physical prototype integration and validation, but at each stage different modelling technology needs exist. The solution hence lies in a scalable approach, involving the combination of multiple and often heterogeneous simulation methods into full system models. It is only in the simulation of the full system behaviour that all interactions between components and subsystems as well as the impact of context, environment and actual use can be adequately taken into account. Multi-physics simulation, combining geometric (3D) as well as functional (1D) models, is the goal. Scalable refers here to the capability that, when for specific parts of the design, more refined models become available; these can be interchanged in the full system model. This means in fact that when started from a concept model, the sub-models can be replaced by more detailed models down in the V-cycle, during the progress of the design. In order to make this happen, tools are required to design system architectures and to provide meta-information to models such as a clear definition of the meaning of the inputs and outputs, description of the meaning of the sub-model, ... to enable automatic system synthesis.

Interoperability not only of software tools, but of modelling concepts is a prerequisite for an integrated mechatronic simulation approach. Important in this discussion is that the final target of the design is in most cases not limited to purely mechanical and/or electrical system, but will involve controls embedded in software. The embedding of control into the design of physical systems is more than just including another discipline into the multi-physics simulation. It is a cornerstone and the start of a new era in modelling and simulation.
Traditionally, the physical system design departments are well separated from the control and software departments, having their own design processes and procedures. Merging and porting concepts, ideas, design processes and procedures from and to both worlds will lead to new design paradigms.

An important pre-requisite for interoperability is a standard to interconnect models, such as the Functional Mock-up Interface (FMI), later explained in the text. The FMI is actually inspired on the concept of Virtual Functional Bus (VFB), used in the AUTOSAR standard [33]. The objective is to make embedded software as hardware independent as possible. This is achieved by a layered structure which separates clearly the software functions on vehicle level, denoted by AUTOSAR software components, from the middle- and firmware (hardware-dependent software parts, which are necessary for the implementation of the functions). Figure 19, from [33], shows the structure of the AUTOSAR architecture. The border or better interface between the hardware-independent software layer and the dependent software layer is denoted by the AUTOSAR Runtime Environment (RTE). The RTE acts like an operating system. Theoretically, it should be possible to easily replace electronic hardware without touching the hardware-independent software components. In this way, the idea of a VFB is created. During the development of software functions on vehicle level, abstraction can be made on how these are mapped on electronic control units or ECUs, how they are connected by vehicle networks or what specific ECU hardware is used. This way, the software components are connected to each other by a virtual bus, called the VFB.

The idea of the VFB is illustrated in Fig. 20, from [33]. By using the AUTOSAR standard during the design of the software functions, complete abstraction is made from the hardware implementation by using the concept of VFB. By defining ECU and system constraint descriptions, the basic software and RTE are configured and mapped automatically on the ECU's. 


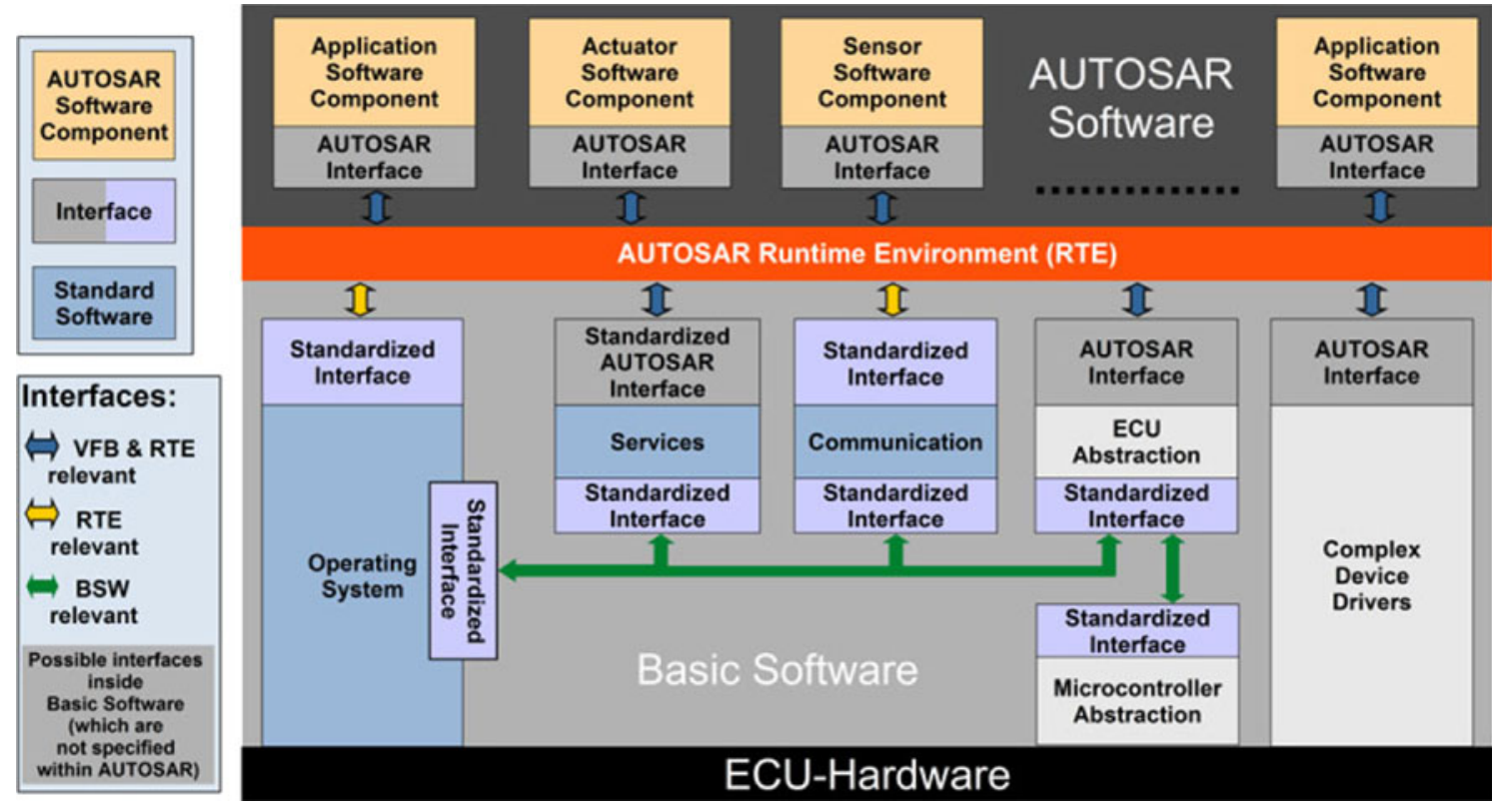

Fig. 19 Layered structure of the AUTOSAR standard [33]

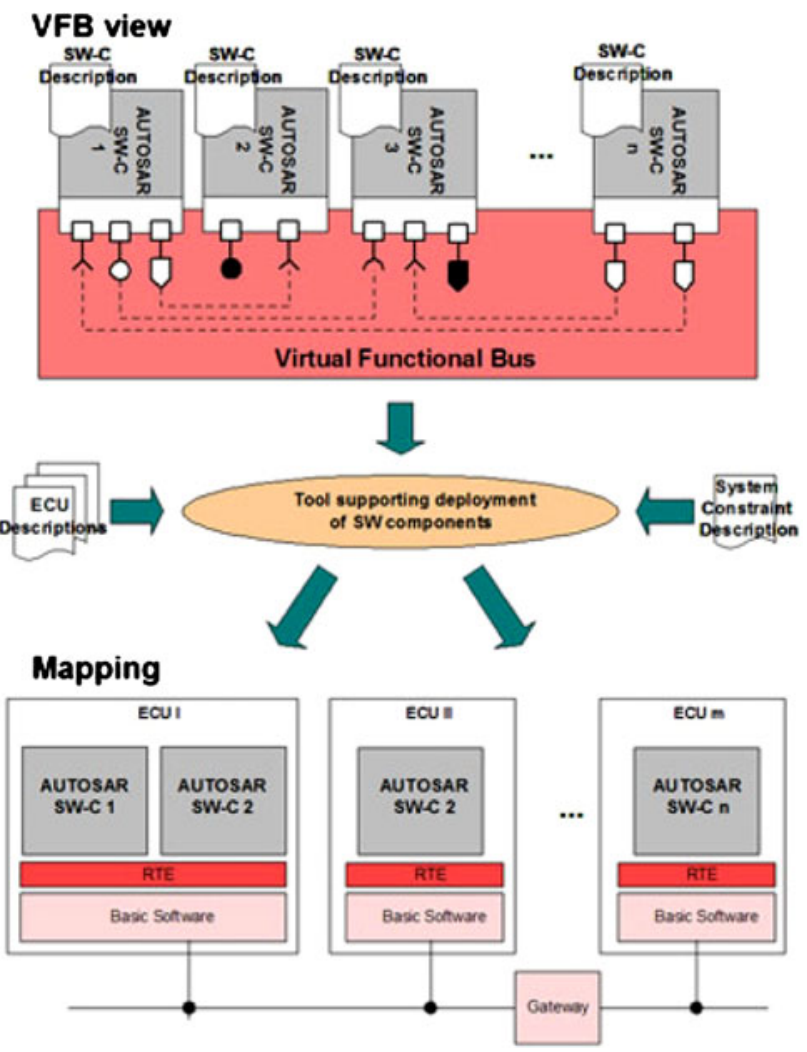

Fig. 20 Virtual Functional Bus (VFB) concept [33]

This evolution will have its impact on the vehicle-level design engineering approach. Due to the inherently multidisciplinary nature of mechatronic systems, different modelling software tools are used. To test, validate, optimize the integrated system before prototypes are made, these different models need to be coupled. Referring to AUTOSAR, the world of the embedded software, the multi-physics models developed in the different software environments take the role of the AUTOSAR software components in Figs. 19 and 20. However, the models run in different programs or even on different computers, have different user interfaces and cannot be coupled as they contain overhead, requiring to run the model on the specific tool. In order to enable to couple the different models, created by different tools, also a layered structure is introduced and the concept of VFB has been taken over. This is performed in a European ITEA2 collaborative research project MODELISAR [47], where the role of VFB is taken up by the Functional Mock-up Interface [48], an open vendor-independent interface (Fig. 21). Unlike suggested by Fig. 21 and the name MODELISAR, the FMI is not restricted to Modelica [49] models. With the current state of the art for coupling multi-physics models, only two or three models can be simulated together as shown in the examples of the paper. When initiatives like the FMI break through, a really large multi-physics simulation can be performed. System integration tests, which are nowadays mostly performed on prototypes, can then be done based on large coupled simulation models.

Another cross fertilization between the physical design world and the world of control and embedded software is the use of models to test embedded software. For example, a virtual car model can be used to create stimuli and feedback for electronic modules. Presently, such models are very basic and, most of the time, implement numerical 
Fig. 21 MODELISAR multifunctional system mock-up

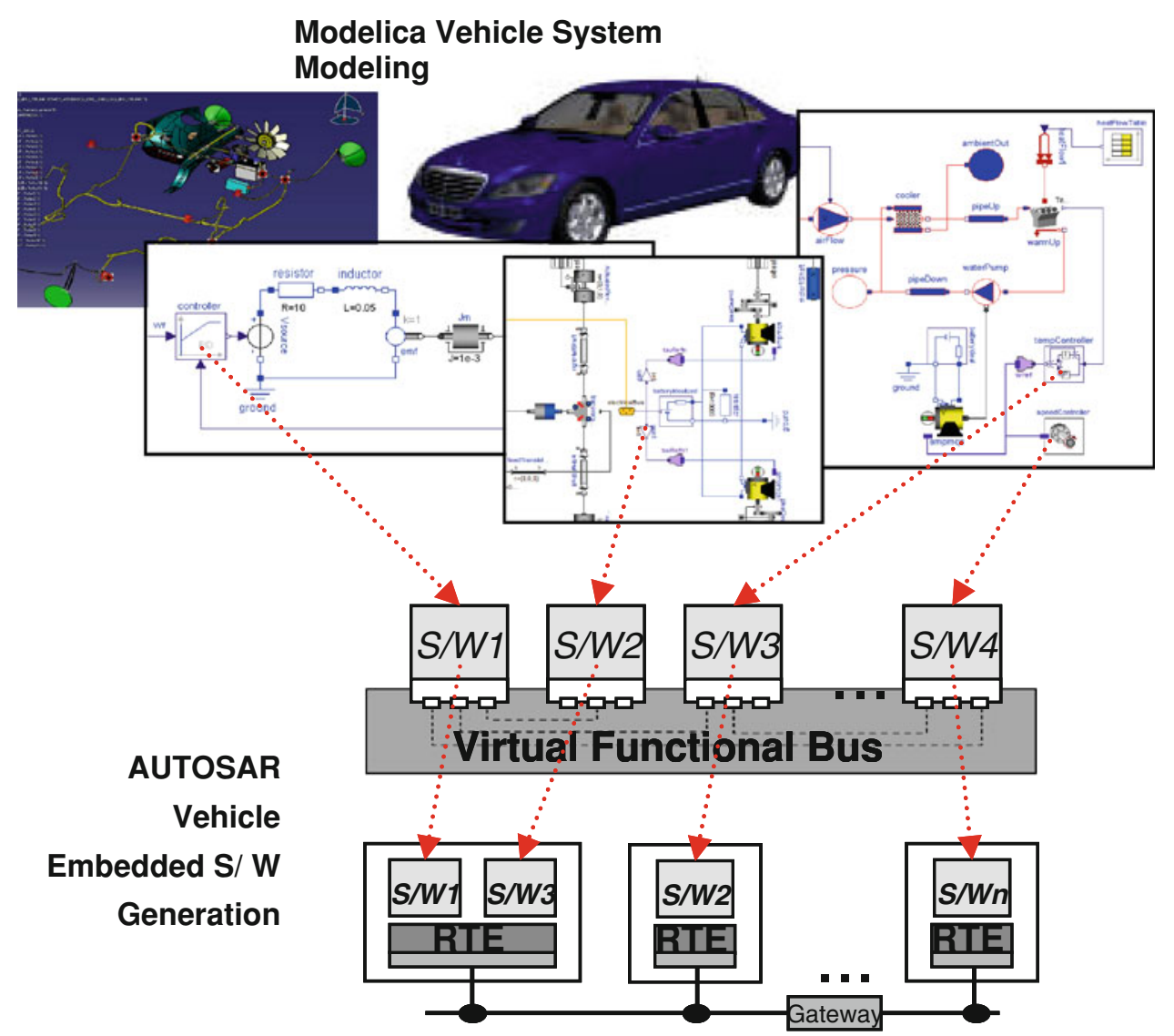

tables instead of physical laws. Additionally, they are created in the software departments where the core knowledge is software engineering and not physical system design. By using the models of the physical design world, a real coupling with software engineering can be achieved. In this way, the double- $\mathrm{V}$ process for mechatronic system design can be achieved as depicted in Fig. 1 and a real mechatronic design can be performed. The physical design and embedded software design process run concurrently such that better integration is achieved. In a very early design phase, model-in-the-loop (MiL) tests can be carried out where software models can be coupled with physical models. As software is tested with physical models, virtual changes to the mechanic, hydraulic or pneumatic design can be performed and more variants can be evaluated. The same holds for software-in-the-loop (SiL) and hardware-inthe-loop (HiL). In the near future, it is expected that by reusing models for physical design for MiL, SiL and HiL, a stronger coupling between the different V-cycles will take place.

The concepts of MiL, SiL and HiL can also be used beyond the control system design in the design of physical systems. This was already illustrated by the example in Sect. 4.3. To make this process more practical, a lot of steps that still are done manually should be automated. The ASAM [50] organization is in this respect a nice initiative to standardize HiL as it works on a uniform standard for data-acquisition systems. Also, the FMI plays an important role to automate the creation of HiL setups for physical component testing by providing standardized interfaces to models and applications that run models. In principle when the HiL process becomes more automated, software models can be easily replaced by hardware components. This is illustrated in Fig. 22 where an ABS/ESP system is designed. The ABS/ESP system needs to interact with the brake system controlling the vehicle, which is operated by a driver. These four components can be modelled. As soon as the physical hardware becomes available, the model can be replaced by the hardware.

In case of Fig. 22, a real person is driving the virtual vehicle, which operates the physical ABS/ESP unit, interacting with a model of the brake hydraulics. Pictures of the setup and screen shots of the model are shown on top in the figure. When HiL systems are used for physical components or system testing, more upfront engineering can be performed and part of the load of the right side of the V-cycle (double V) will be transferred to the left side of the $\mathrm{V}$. This is beneficial as the cost for solving problems is much higher on the right side of the $\mathrm{V}$.

To make the ideas of Fig. 22 common practice, models should be easily replaceable. In the early design cycle, the vehicle model can be just a simple mass with some rolling 

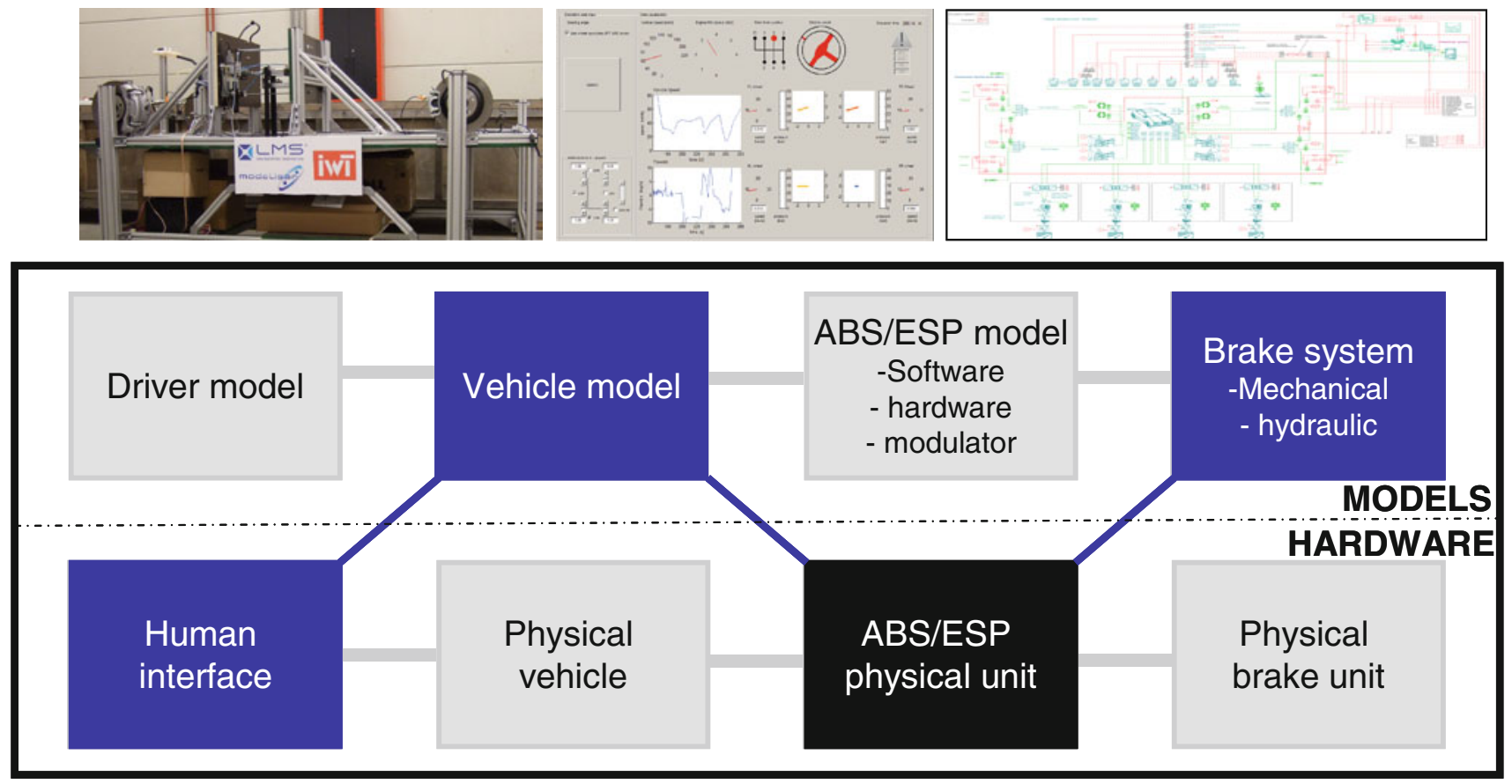

Fig. 22 Standardized interphases will allow to easily switch between models and hardware components

resistance and wind loads model. During the design cycle, more information becomes available and there is a need to replace the simple vehicle model by a multi-body model. To make this work, a standard like the FMI is not sufficient. An additional layer should be included, defining the structure of models, the type and kind of inputs and outputs and the units. Therefore, a new vendor-independent standard is required to specify this meta data. By this, the design process is more automated and more important decisions are frontloaded.

By merging the physical design cycle with the embedded software design cycle, more concurrent engineering will be possible. Nowadays, in the car industry, the design is driven by mechanics in the form of geometries. When the geometry is fixed, packaging studies are performed to allocate space to actuators and sensors such that these subsystems can be designed. Afterwards, embedded software development can take place. Driven by the need to come to really integrated systems, which is actually the true definition of a mechatronic system, a new design paradigm is required.

Design concepts originating from software design, often referred to as model-driven engineering, seem to provide an answer. A typical software design cycle starts from requirements. Once the requirements are fixed, the system architecture is defined and a concept model can be created. Then software modules are split into components and elaborated in more detail. These ideas are transferred to the automotive industry and are called model-based system engineering (MBSE) [5-9]. However, in order to really follow such a structured approach, some new challenges need to be tackled.

A first challenge lies at the border of system requirements, system architecture and concept model phase. To cross this border, a detailed analysis of the machine behaviour, detailed component and subsystem models are needed. However, early in the design phase, detailed solutions are not available yet; only the requirements are known, containing a problem description and a design space, limited by a number of constraints. Nowadays, the concept solution or solution architecture is decided upon by the core design team in a process that is only to a very limited degree supported by tools of any kind. It is, however, expected that a systematic (formal) description of the machine requirements, on the one hand, and solution concepts, on the other, will allow exploiting the tremendous calculation capabilities of present day computers to assist the designer in the generation and the evaluation of the performance of different concepts meeting the specified requirements. Extending the above-mentioned, multidomain mechatronic simulation environment with such synthesis capabilities, next to analyses capabilities, would allow further reducing the duration and cost of the design cycle dramatically.

Another challenge lies in automatic model generation and updating. Essentially, the requirements capture a description of the system. The models, created by engineers to analyse and design the system, should meet the requirements and are in that respect a different formulation of the requirements. This implies that somehow, to a 
certain extent, models, as also in the software world to some extent software code, can be generated automatically. It is clear that this process can only be achieved partially, as during the design process additional decisions need to be taken on lower levels. Therefore, higher-level models inherently rely on assumptions, creating the need to automatically update higher-level models once lower-level models are developed. For example, a stiffness value in a concept phase design model could result in a relation between two points on a structural finite element grid. By translation of information of lower-level design models to higher-level system parameters, assumptions can be verified.

One may conclude that a lot of evolution is taking place in the design of mechatronic systems. If the evolution continues and solutions to the highlighted challenges can be solved, the design cycles in the mechatronics world will radically change in the coming years.

\section{Conclusion}

The performance engineering of mechatronic vehicle systems mandates simulation and test methods that are capable of simulating, analysing and optimizing the performance of such a product, taking into account the interaction of many subsystems as well as the environment and working as active systems with sensors, actuators and interconnections to controllers.

The key to any hereto applicable virtual testing approach is the combined simulation of multi-physics systems and controls. The actual integration between system models and control models depends on the purpose of the analysis: systems engineering or controller design and optimization. Depending on the phase of the design process, model-in-the-loop, software-in-the-loop and hardware-in-the-loop approaches can be distinguished, each with their own rationale for model integration, real-time performance and hardware connection. To make this process effective and efficient, a scalable multi-physics and control integration approach is proposed, based on a seamless integration of 3D, 1D and control models, starting from requirement modelling and ending with product validation. This paves the way to a model-based system engineering approach as known from the software industry.

\footnotetext{
Acknowledgments The presented research is conducted in the context of a coordinated research effort for the development of design engineering solutions for mechatronic system. This programme is cofunded through a number of research projects such as ITEA2 projects Modelisar and Modrio and Flanders' Drive projects ReVAS (Research Vehicle for Active Safety) and InAst (Integrated Active Safety Technology), supported by IWT Vlaanderen and the Marie Curie projects Smart Structures CAE, and VECOM, co-financed by
}

the European Commission. The authors acknowledge the contribution of many colleagues and partners involved in these projects, in particular Dr. Leopoldo de Oliveira from University Sao Paulo in Brazil and the Marie Curie fellows Marco Gubitosa and Ondrej Marek hosted at LMS. The active suspension research is conducted in close cooperation with Flanders' Drive and the companies Tenneco Automotive, Verhaert and Triphase. The active noise control research was performed in close cooperation with the research group PMA at the KU Leuven and the Fraunhofer Institut LBF in Darmstadt. All their inputs are gratefully acknowledged.

Open Access This article is distributed under the terms of the Creative Commons Attribution License which permits any use, distribution, and reproduction in any medium, provided the original author(s) and the source are credited.

\section{References}

1. Schramm D, Lalo W, Unterreiner M (2010) Application of simulators and simulation tools for the functional design of mechatronic systems. Robot Autom Syst 166-167:1-14

2. Van Beek TJ, Tomiyama T (2008) Connecting views in mechatronic systems design, a function modeling approach. In: Proceedings of 2008 IEEE/ASME international conference on mechatronic and embedded systems and applications, Oct. 12-15, 2008, Beijing, China, pp 164-169

3. Cabrera A, Woestenenk K (2011) An architecture model to support cooperative design for mechatronic products: a control design case. Mechatronics 21(3):534-547

4. Cabrera A, Foeken MJ, Tekin OA, Woestenenk K, Erden MS, De Schutter B, Van Tooren MJL, Babuska R, van Houten FJ, Tomiyama T (2010) Towards automation of control software: a review of challenges in mechatronic design. Mechatronics 20(8):876-886

5. Brown AW (2004) Model driven architecture: principles and practice. Softw Syst Model (SoSym) 3:314-327

6. Mosterman PJ, Vangheluwe H (2004) Computer automated paradigm modelling: an introduction. Simulation 80(9):433-450

7. Huining Feng T, Zia M, Vangheluwe H (2007) Multi-formalism modelling and model transformation for the design of reactive systems. In: Summer computer simulation conference (SCSC 2007), July 2007, San Diego, CA, USA, pp 505-512

8. Syed F, Nallapa R, Ramaswamy D (2007) Integrated modeling environment for detailed algorithm design, simulation and code generation. In: Proceedings of SAE world congress \& exhibition, April 2007, Detroit (MI-USA). SAE Paper 2007-01-0274

9. Warwick G, Norris G (2010) Designs for success, systems engineering must be rethought if program performance is to improve. Aviation Week Space Technol 172(40):72-75

10. Valsan A (2006) Trends, technology roadmaps and strategic market analysis of vehicle safety systems in europe. International automotive electronics congress, Paris, France, Oct. 24, 2006

11. McKinsey's automotive \& assembly practice (2004) HAWK 2015-Knowledge-based changes in the automotive value chain, McKinsey Brochures

12. JSAE (2007) The automobile in the year 2030, society of automotive engineers of Japan

13. System design: new product development for mechatronics, Aberdeen Group, http://www.aberdeen.com

14. Laurgeau C (2008) Present and future of intelligent transportation systems. In: Proceedings of ICAT 2008, international conference on automotive technologies, Nov. 13-14, 2008, Istanbul, Turkey 
15. Vahidi A et al (2003) Research advances in intelligent collision avoidance and adaptive cruise control. IEEE Trans Intell Transp Syst 4(3): 143-153

16. European Commission (2007) Towards Europe-wide safer, cleaner and efficient mobility: the first intelligent car report. Communication from the Commission to the European Parliament, the Council, the European Economic and Social Committee and the Committee of the Regions, COM (2007) 541, 17.09.2007

17. Costlow $T$ (2008) Managing software growth. Automotive Engineering International, Nov. 20, 2008

18. Van der Auweraer H, Vecchio A, Peeters B, Dom S, Mas P (2008) Hybrid testing in aerospace and ground vehicle development. In: Mettupalayam S, Saouma V (eds) Hybrid simulation: theory, implementation and applications, Taylor \& Francis Group, London, pp 203-214

19. Plateaux R, Choley JY, Penas O, Riviere A (2009) Towards an integrated mechatronic design process. In: Proceedings of IEEE ICM international conference on mechatronics, Apr. 14-17, 2009, Malaga, Spain, pp 114-119

20. Plateaux R, Penas O, Choley JY, M'henni F, Riviere A (2010) Integrated design methodology of a mechatronic system. Mécanique Ind 11(5):401-406

21. Forsberg K, Mooz H (1991) The relationship of systems engineering to the project cycle. 1st annual symposium of the national council on systems engineering (NCOSE), October 1991

22. Eichberger A, Rulka W (2004) Process save reduction by macro joint approach: the key to real time and efficient vehicle simulation. Veh Syst Dyn 41(5):401-413

23. Fujimoto RM (2003) Distributed simulation systems. In: Proceedings of 2003 winter simulation conference IEEE, New Orleans, LA, USA

24. González F, Naya MA, Luaces A, González M (2011) On the effect of multirate co-simulation techniques in the efficiency and accuracy of multibody system dynamics. Multibody Syst Dyn 25(4):461-483. doi:10.1007/s11044-010-9234-7

25. Balboni A, Fornaciari W, Sciuto D (1996) Co-synthesis and cosimulation of control-dominated embedded systems. Des Autom Embed Syst 3(1):257-289

26. Guyan R (1965) Reduction of mass and stiffness matrices. AIAA $\mathrm{J}$ 3:380

27. Craig RJ (1987) A review of time-domain and frequency domain component mode synthesis methods. Int J Anal Exp Modal Anal 2(2):59-72

28. Antoulas AC, Sorensen DC (2001) Approximation of large-scale dynamical systems: an overview. Int J Appl Math Comp Sci 11:1093-1121

29. Balmes E (1996) Parametric families of reduced finite element models. Theory and applications. Mech Syst Signal Process 10(4):381-394

30. Bruls O, Duysinx P, Golinval JC (2007) The global modal parameterization for non-linear model-order reduction in flexible multibody dynamics. Int J Numer Meth Eng 69:948-977

31. Ciminello M, Ameduri S, Concilio A (2008) FE modelling of an innovative vibration control shunt technique. J Intell Mater Syst Struct 19(8):875-887

32. Bacic M (2005) On hardware-in-the-loop simulation. In: Proceedings of the 44th IEEE conference on decision and control, and the European control conference 2005 Seville, Spain, December 12-15, 2005

33. http://www.autosar.org/

34. Kvasnicka P, Prokop G, Rettinger A, Stahl H (2006) Comprehensive approach to the chassis control development. In: Proceedings of SAE 2006 world congress \& exhibition, April 3-6, 2006, Cobo Center, Detroit, MI, USA. SAE Paper 2006-01-1280

35. Prescott WC, Laughlin JA (2007) Implementation and usage of design sensitivity analysis in multibody dynamics software. In: Proceedings of eccomas, thematic conference on multibody dynamics, Milan, Italy, June 2007

36. Gubitosa M, De Cuyper J, Cibrario V (2008) Optimized design method of vehicle suspension systems using a reverse engineering approach. In: Proceedings of IMECE 2008, Nov. 2-6, 2008, Boston (MA-USA). Paper IMECE2008-66623

37. De Cuyper J, Gubitosa M, Kang J, Lethé G, Furmann M, Kading D (2008) Vehicle dynamics control-a case study in LMS Virtual.Lab Motion. In: Proceedings of 4th Asian conference on multibody dynamics 2008, Aug. 20-23, 2008, Seogwipo-city, Korea

38. Alirand M, Botelle E, Sau J (1999) Modeling a force control actuator for semi active car dampers- basics. 16th IAVSD symposium, dynamics of vehicles on roads and tracks, Aug 29-Sept03 1999, Pretoria, South Africa

39. He Y, McPhee J (2005) Multidisciplinary design optimization of mechatronic vehicles with active suspensions. J Sound Vib 283:217-241

40. Lauwerys C, Swevers J, Sas P (2005) Robust linear control of an active suspension on a quarter car test-rig. Control Eng Pract 13:577-586

41. Swevers J, Lauwerys C, Vandersmissen B, Maes M, Reybrouck K, Sas P (2007) A model-free control structure for the on-line tuning of the semi-active suspension of a passenger car. Mech Syst Signal Process 21:1422-1436

42. Van der Auweraer H, Mas P, Segaert P, de Oliveira L, da Silva M, Desmet W (2007) CAE-based design of active noise control solutions. In: Proceedings of SIAT 2007, Pune (India), 17-20 Jan. 2007. SAE Paper 2007-26-032

43. de Oliveira LPR, da Silva MM, Van Brussel H, Sas P, Desmet W (2008) Concurrent mechatronic design approach for active control of cavity noise. J Sound Vib 314:507-525

44. Mohring J, Wirsen A (2006) Robust controller design based on integrated simulation of an ANVC-System. In: Proceedings of Euronoise 2006, Tampere, Finland, May 30-June 1, 2006

45. Herold S, Atzrodt H, Mayer D, Thomaier M (2005) Integration of different approaches to simulate active structures for automotive applications. In: Proceedings of Forum Acusticum 2005, Budapest, Hungary, Aug. 29-Sep. 2, 2005

46. de Oliveira LPR, Janssens K, Gajdatsy P, Van der Auweraer H, Varoto PS, Sas P, Desmet W (2008) Active sound quality control of engine induced cavity noise. Mech Syst Signal Process 23:476-488

47. Bichet F (ed) (2007) MODELISAR, From system modeling to $\mathrm{S} / \mathrm{W}$ running on the vehicle. ITEA2 full project proposal

48. http://functional-mockup-interface.org/

49. http://www.modelica.org/

50. http://www.asam.net/ 